\title{
Si nanocrystal-based LEDs fabricated by ion implantation and plasma-enhanced chemical vapour deposition
}

\author{
M. Perálvarez ${ }^{1}$, J. Barreto ${ }^{2}$, Josep Carreras ${ }^{1}$, A. Morales ${ }^{2}$, D. \\ Navarro-Urrios $^{1}$, Y. Lebour ${ }^{1}$, C. Domínguez ${ }^{2}$ and B. Garrido ${ }^{1}$ \\ ${ }^{1}$ IN $^{2}$ UB-EME, University of Barcelona. C/Marti i Franques 1, PL2, 08028 \\ Barcelona, Spain \\ 2 IMB-CNM, CSIC, Bellaterra 08193, Barcelona, Spain \\ E-mail: mperalvarez@el.ub.es
}

\begin{abstract}
An in-depth study of the physical and electrical properties of Sinanocrystals embedded in silicon dioxide is presented. These layers were fabricated with different $\mathrm{Si}$ concentrations by both ion implantation and plasma-enhanced chemical vapour deposition. Subsequently, LEDs devices based on a metal-oxide-silicon configuration with a $\sim 350 \mathrm{~nm}$ polycrystalline $\mathrm{Si}$ top electrode and an active layer of about $45-50 \mathrm{~nm}$, were fabricated in conventional lithography process. In order to optimize the device performances, prior to the top electrode deposition, the structural and photoluminescent properties of the active layers were exhaustively studied.

Devices fabricated by ion implantation exhibit a combination of direct current and field-effect luminescence under a bipolar pulsed voltages excitation. The onset of the emission decreases with the $\mathrm{Si}$ excess from 6 to $3 \mathrm{~V}$. The direct current emission is attributed to impact ionization, and is associated with the reasonably high current levels observed in current-voltage measurements. This behaviour is in good agreement with transmission electron microscopy images that revealed a continuous and uniform Si-nanocrystals distribution. The emission power efficiency is relatively low, $\sim 10^{-3} \%$, and the emission intensity exhibits fast degradation rates, as revealed from accelerated aging experiments.

Devices fabricated by chemical deposition only exhibit field-effect luminescence which onset decreases with the Si excess from 20 to $6 \mathrm{~V}$. The absence of the continuous emission is explained by the observation of a 5 -nm region free of nanocrystals, which strongly reduces the direct current through the gate. The main benefit of having this nanocrystal-free region is that tunnelling current flow assisted by nanocrystals is blocked by the $\mathrm{SiO}_{2}$ stack so that power consumption is strongly reduced, which in return increases the device power efficiency up to $0.1 \%$. In addition, the accelerated aging studies reveal a $50 \%$ degradation rate reduction as compared to implanted structures.
\end{abstract}

PACS numbers: 73.63.Bd, 78.67.Bf, 85.60.Jb

Submitted to: Nanotechnology 


\section{Introduction}

Monolithic silicon light emitting diodes (LEDs) and lasers are expected to dramatically evolve silicon technology, by opening new areas of application for Si never thought possible in the past $[1,2,3,4,5,6,7]$. In indirect gap semiconductors such as $\mathrm{Si}$ the role of the radiative exciton recombination across the band-gap is very low, since it is required the participation of a phonon, which makes it a second order process with low quantum efficiency. With the aim to improve the radiative recombination rate in $\mathrm{Si}, \mathrm{Si}$ nanocrystals (Si-nc) have been object of extensive research since the electron and hole wavefunctions are confined into a small volume. Nowadays, systems composed of Si-nc embedded in $\mathrm{SiO}_{2}$ (silicon dioxide) represent a promising alternative thanks to their chemical stability and their strong and tunable emission in the red-infrared range (from 1.4 to $1.7 \mathrm{eV})$.

A wide variety of techniques are used for the fabrication of $\mathrm{Si}-\mathrm{nc} / \mathrm{SiO}_{2}$ layers, all of them based on first preparing silicon-rich-oxide (SRO) layers and anneal in order to propitiate the Si-nc growth. Ion implantation and plasma-enhanced chemical vapour deposition (PECVD) are two of the most frequently reported. In the former, the excess of silicon is generated by implantation of $\mathrm{Si}$ atoms into thermally grown oxide. This technique provides an accurate method to control the silicon profile as the $\mathrm{Si}$ dose and implantation energy can be precisely monitored. However, ion implantation presents two main drawbacks: i) the impact of the incident ions on the host matrix generates an undesired concentration of defects, and ii) the achievement of uniform $\mathrm{Si}$ profiles in thick oxide layers is limited by the energy required in order to perform deep implantation processes. By PECVD, the fabrication of thick SRO layers is possible. The plasma enhanced process stands out against the different deposition techniques as thicker layers can be rapidly grown at low temperatures. However, as in the case of ion implantation technique, the concentration of defects must be controlled, a process that strongly depends on the deposition rate.

In recent years, the emission from Si-nc based materials under electrical pumping has been widely reported $[8,9,10,11]$, mostly under DC (direct current) polarization. The application of relatively high voltages under DC regime may compromise the reliability of the device due to the high currents involved which result in fast degradation rates. In thinner devices, this degradation can be overcome, but at the cost of its emission efficiency, as the recombination of electrons and holes injected from opposite electrodes is possible but not effective due to the increase of the continuous leakage current between the electrodes. Latest studies have demonstrated electroluminescence (EL) emission under alternate polarization applying the concept of field-effect luminescence[12, 13]. This mechanism, which consists of sequential injection of electrons and holes from the substrate, optimizes the device electrical stress and reduces the power consumption, leading to higher emission efficiencies.

In this work, we firstly present an exhaustive study on the structural properties of SRO layers fabricated by PECVD and ion implantation, establishing a correlation 
with the characteristics of the emission under optical excitation. The results of this analysis are then compared with the electrical and electro-optical properties of LEDs fabricated under similar conditions. The field effect luminescence is studied in-depth, paying special attention to the different behaviours observed in samples fabricated by these two elaboration techniques .

\section{Experimental}

\begin{tabular}{ccccccccc}
\hline & & & \multicolumn{5}{c}{ Thickness (nm) } \\
\cline { 3 - 8 } Sample & $\begin{array}{c}{\left[\mathbf{N}_{\mathbf{2}} \mathbf{O}\right] /\left[\mathrm{SiH}_{4}\right]} \\
\text { Ratio }\end{array}$ & \multicolumn{2}{c}{ Si excess (at. \%) } & $\begin{array}{c}\text { As- } \\
\text { deposited }\end{array}$ & Annealed \\
\hline P1 & 22.00 & 5.39 & 1.64 & 107 & 4 & 102 & 4 \\
\hline P2 & 9.17 & 10.92 & 1.41 & 110 & 4 & 88 & 1 \\
\hline P3 & 5.50 & 16.94 & 1.37 & 93 & 5 & 74 & 3 \\
\hline P4 & 3.60 & 26.10 & 1.38 & 105 & 3 & 85 & 2 \\
\hline P5 & 2.20 & 42.06 & 1.33 & 33 & 10 & 22 & 4 \\
\hline
\end{tabular}

Table 1. PECVD samples

Two sets of SRO layers were fabricated onto a p-type Si substrate (resistivity of 0.1-1.4 $\Omega \cdot \mathrm{cm}$ ) by PECVD and ion implantation with different Si excesses. Their characteristics are detailed in Tables 1 for PECVD and 2 for implanted films, respectively. The samples are referred by P for PECVD and I for ion-implanted. A clear definition of the Si excess can be found in reference[14]. Deposited layers were fabricated with silane $\left(\mathrm{SiH}_{4}\right)$ and nitrous oxide $\left(\mathrm{N}_{2} \mathrm{O}\right)$ as source gases, by keeping constant the $\mathrm{N}_{2} \mathrm{O}$ flow and adjusting the $\mathrm{N}_{2} \mathrm{O} / \mathrm{SiH}_{4}$ ratio to the required $\mathrm{Si}$ contents. More details on the fabrication process are given elsewhere[15]. Silicon nanocrystal formation and phase separation were achieved by high temperature annealing to $1250{ }^{\circ} \mathrm{C}$ in a $\mathrm{N}_{2}$ atmosphere for $1 \mathrm{~h}$. The implanted layers were fabricated by implantation of silicon into thermally grown silicon oxide in a two-step process (energies of $25 \mathrm{keV}$ and $50 \mathrm{keV}$ ). In order to obtain and in-depth flat profile of $\mathrm{Si}$, a $\sim 30 \mathrm{~nm}$-thick buffer layer of $\mathrm{Si}_{3} \mathrm{~N}_{4}$ (silicon nitride) was deposited by PECVD onto the oxide layer before the implantation. The results of the implantation process were previously simulated by SRIM (stopping and range of ions in matter) calculations[16]. The phase separation and growth of the Si-nc was achieved by submitting the SRO layers to high temperature annealing at $1100{ }^{\circ} \mathrm{C}$ in $\mathrm{N}_{2}$ atmosphere for $4 \mathrm{~h}$.

Different techniques were used for the characterization of the fabricated layers. Ellipsometry measurements were performed to evaluate the thickness and the refractive index of the different active layers using a Rudolph Research AutoEl IV ellipsometer, operating at $632.8 \mathrm{~nm}$ under an incidence angle of $70^{\circ}$. XPS (X-ray photoelectron spectroscopy) measurements were performed in order to study the composition profiles of the prepared layers. The signature of the vibronic modes of the 


\begin{tabular}{|c|c|c|c|c|c|c|c|c|}
\hline \multirow{2}{*}{ Sample } & \multirow{2}{*}{$\begin{array}{c}\text { Implanted dose } \\
\left(\mathrm{cm}^{-2}\right)\end{array}$} & \multirow{2}{*}{$\begin{array}{c}\text { Ion energy } \\
(\mathrm{keV})\end{array}$} & \multirow{2}{*}{\multicolumn{2}{|c|}{$\begin{array}{l}\text { Si excess } \\
\text { (at. \%) }\end{array}$}} & \multicolumn{4}{|c|}{ Thickness (nm) } \\
\hline & & & & & $\overline{\mathrm{Si}_{3}}$ & & $\overline{\mathrm{SR}}$ & \\
\hline \multirow{2}{*}{ I1 } & $6.0 \cdot 10^{15}$ & 25 & \multirow{2}{*}{7.45} & \multirow{2}{*}{1.68} & \multirow{2}{*}{17.0} & \multirow{2}{*}{1.0} & \multirow{2}{*}{42.4} & \multirow{2}{*}{0.8} \\
\hline & $2.4 \cdot 10^{16}$ & 50 & & & & & & \\
\hline \multirow{2}{*}{ I2 } & $1.2 \cdot 10^{16}$ & 25 & \multirow{2}{*}{9.37} & \multirow{2}{*}{1.63} & \multirow{2}{*}{18.3} & \multirow{2}{*}{0.4} & \multirow{2}{*}{65.5} & \multirow{2}{*}{0.4} \\
\hline & $4.8 \cdot 10^{16}$ & 50 & & & & & & \\
\hline \multirow{2}{*}{ I3 } & $1.8 \cdot 10^{16}$ & 25 & \multirow{2}{*}{11.21} & \multirow{2}{*}{1.55} & \multirow{2}{*}{20.0} & \multirow{2}{*}{4.0} & \multirow{2}{*}{60.0} & \multirow{2}{*}{3.0} \\
\hline & $7.2 \cdot 10^{16}$ & 50 & & & & & & \\
\hline \multirow{2}{*}{ I4 } & $2.4 \cdot 10^{16}$ & 25 & \multirow{2}{*}{14.25} & \multirow{2}{*}{1.50} & \multirow{2}{*}{21.0} & \multirow{2}{*}{1.0} & \multirow{2}{*}{42.0} & \multirow{2}{*}{1.0} \\
\hline & $9.6 \cdot 10^{16}$ & 50 & & & & & & \\
\hline
\end{tabular}

Table 2. Ion-implanted samples

matrix was measured by FTIR (Fourier transform infra-red) in a Perkin Elmer 2000 spectrophotometer. The cross-section images of the samples and the size distribution of Si-nanocrystals were obtained by EFTEM (energy-filtered transmission electron microcopy). Photoluminescence (PL) spectra were measured by exciting the samples with a He-Cd laser at $325 \mathrm{~nm}$ and $17 \mathrm{~mW}$. The detection set-up was composed of a $0.6 \mathrm{~m}$ monochromator, a GaAs photomultiplier for measurements in the visible and a Germanium detector for measurements in the NIR (near infra-red) wavelength range. To minimize noise effects, a standard chopper lock-in technique was used. The PL spectra were corrected for the system response, measured with a calibrated tungsten lamp. The PL emission as a function of the incident photon flux and pumping energy was characterized using an $\mathrm{Ar}^{+}$multiline laser. In order to perform time-resolved measurements the $457 \mathrm{~nm}$ and $488 \mathrm{~nm} \mathrm{Ar}^{+}$lines were modulated with a Brimrose FQM-80-2 acousto-optic modulator with $\sim 0.195 \mu$ s characteristic rise-time. In this case, the emission transients were detected at different wavelengths (selected by means of a $1 / 8 \mathrm{~m}$ Cornerstone 130 monochromator) with a photon-counting set-up, composed of a Hamamatsu thermoelectrically cooled H7422-50 photomultiplier and a Stanford Research SR430 Multi-channel scaler/averager.

\begin{tabular}{|c|c|c|c|c|c|c|c|c|}
\hline Sample & $\begin{array}{c}{\left[\mathrm{N}_{2} \mathrm{O}\right] /\left[\mathrm{SiH}_{4}\right]} \\
\text { Ratio }\end{array}$ & $\begin{array}{c}\mathrm{Si} \\
\text { excess } \\
\text { (at. \%) }\end{array}$ & $\begin{array}{c}\text { Thickness } \\
\text { (nm) }\end{array}$ & $\begin{array}{l}\mathrm{C}_{\max } \\
(\mathrm{pF})\end{array}$ & & $\varepsilon_{\mathrm{r}}$ & $\begin{array}{c}\text { Density of } \\
\text { trapped } \\
\text { charge, } N_{t} \\
\left(\mathrm{~cm}^{-2}\right)\end{array}$ & $\begin{array}{c}\text { Charged } \\
\text { nanocrystals } \\
(\%)\end{array}$ \\
\hline PD1 & - & 0 & $41.1 \quad 0.3$ & 209.83 & 4.235 & 0.029 & $1.654 \times 10^{9}$ & -..-- \\
\hline PD2 & 13 & 7.9 & $45 \quad 4$ & 239.85 & 5.300 & 0.451 & $1.438 \times 10^{10}$ & 0.4 \\
\hline PD3 & 10 & 9.9 & $44 \quad 5$ & 248.18 & 5.362 & 0.576 & $3.517 \times 10^{11}$ & 8.9 \\
\hline PD4 & 7 & 13.7 & $\begin{array}{ll}48 \quad 9\end{array}$ & 281.67 & 6.639 & 1.160 & $3.681 \times 10^{12}$ & 85.2 \\
\hline PD5 & 5 & 18.8 & $47 \quad 6$ & 297.24 & 6.862 & 0.890 & $3.835 \times 10^{12}$ & 90.7 \\
\hline
\end{tabular}

Table 3. MOS devices (PECVD) 


\begin{tabular}{|c|c|c|c|c|c|c|c|c|c|}
\hline Sample & $\begin{array}{l}\text { Implanted } \\
\text { dose }\left(\mathrm{cm}^{-2}\right)\end{array}$ & $\begin{array}{c}\text { Ion } \\
\text { energy } \\
\text { (keV) }\end{array}$ & $\begin{array}{c}\mathrm{Si} \\
\text { excess } \\
\text { (at. \%) }\end{array}$ & $\begin{array}{c}\text { Thickness } \\
\text { (nm) }\end{array}$ & $\begin{array}{l}\mathbf{C}_{\max } \\
(\mathbf{p F})\end{array}$ & & $\varepsilon_{\mathrm{r}}$ & $\begin{array}{c}\text { Density of } \\
\text { trapped } \\
\text { charge, } N_{t} \\
\left(\mathrm{~cm}^{-2}\right)\end{array}$ & $\begin{array}{c}\begin{array}{c}\text { Charged } \\
\text { nanocrystals } \\
(\%)\end{array} \\
\end{array}$ \\
\hline ID1 & 0 & 0 & 0 & $\begin{array}{ll}41.1 & 0.3\end{array}$ & 215.51 & 4.342 & 0.032 & $8.456 \times 10^{8}$ & -ב-די-ד \\
\hline ID2 & $\frac{1.2 \cdot 10^{16}}{5.0 \cdot 10^{16}}$ & $\begin{array}{l}25 \\
50\end{array}$ & 9.6 & $\begin{array}{ll}46.3 & 0.5\end{array}$ & 277.88 & 6.307 & 0.068 & $1.199 \times 10^{12}$ & 16.2 \\
\hline ID3 & $\frac{1.4 \cdot 10^{16}}{6.0 \cdot 10^{16}}$ & $\begin{array}{l}25 \\
50\end{array}$ & 10.5 & $\begin{array}{ll}48.8 & 0.2\end{array}$ & 294.78 & 7.052 & 0.029 & $1.509 \times 10^{12}$ & 19.3 \\
\hline ID4 & $\begin{array}{l}1.8 \cdot 10^{16} \\
7.2 \cdot 10^{16}\end{array}$ & $\begin{array}{l}25 \\
50\end{array}$ & 11.7 & $47 \quad 3$ & 320.51 & 7.384 & 0.471 & $9.017 \times 10^{11}$ & 12.0 \\
\hline ID5 & $\frac{1.8 \cdot 10^{16}}{7.2 \cdot 10^{16}}$ & $\begin{array}{l}25 \\
50\end{array}$ & 11.7 & $\begin{array}{ll}46 \quad 3 \\
\end{array}$ & 308.60 & 6.959 & 0.454 & $4.167 \times 10^{11}$ & 5.7 \\
\hline ID6 & $\frac{2.0 \cdot 10^{16}}{8.3 \cdot 10^{16}}$ & $\begin{array}{l}25 \\
50\end{array}$ & 12.6 & $50 \quad 4$ & 315.74 & 7.739 & 0.619 & $3.558 \times 10^{11}$ & 4.4 \\
\hline
\end{tabular}

Table 4. MOS devices (ion-implanted)

In the next stage, Si-nc based metal-oxide semiconductor (MOS) structures were fabricated and characterized. Tables 3 and 4 present some experimental data of PECVD and implanted devices, respectively. PECVD devices are labelled as PD and implanted ones are labelled as ID. In order to establish a reliable comparison, deposited and implanted structures were prepared with similar thickness about 45-50 nm. N-doped semitransparent polycrystalline silicon (poly-Si) 350 nm-thick was deposited as a top contact layer and aluminium pads were placed to facilitate the probe connection. Before this step and only for the implanted structures, the nitride buffer was chemically etched. Even though the devices were prepared with different contact areas (see figure 12), only those with larger electrodes areas were studied $\left(2.3 \times 10^{-3}\right.$ and $\left.9.6 \times 10^{-3} \mathrm{~cm}^{2}\right)$, since they exhibit the best signal-to-noise ratio of EL. In order to characterize the influence of the top electrode on the luminescence, transmittance spectra of the polycrystalline silicon layers were measured. To do so, poly-Si layers were deposited onto fused silica substrates and optical transmission measurements were performed with a Shimadzu UV-2101 PC spectrometer. The EL data were taken by polarizing with variable width and frequency bipolar square waves from an Agilent 8114A Pulse Generator. Timeresolved electroluminescence was detected with the photon-counting set-up described above. The EL spectra were measured by means of a Princeton Instruments 100B LN (liquid nitrogen) cooled CCD camera and an Oriel MS257 1/4 m Monochromator. Quasi-static I-V (current-voltage) and C-V (capacitance-voltage) characteristics were measured using an Agilent B1500 semiconductor device analyzer.

\section{Structural characterization}

\section{1. $P C V D$}

Table 1 shows, in addition to the nominal $\left[\mathrm{N}_{2} \mathrm{O}\right] /\left[\mathrm{SiH}_{4}\right]$ ratio and the $\mathrm{Si}$ excess yield by XPS measurements, the thickness of as-deposited and annealed PECVD layers measured by ellipsometry. After the high temperature annealing $\left(1250{ }^{\circ} \mathrm{C}\right)$, the ellipsometry measurements revealed an important reduction in the layer thickness due to $\mathrm{H}$ desorption and atomic redistribution. This compaction has also been reported in reference [17]. 


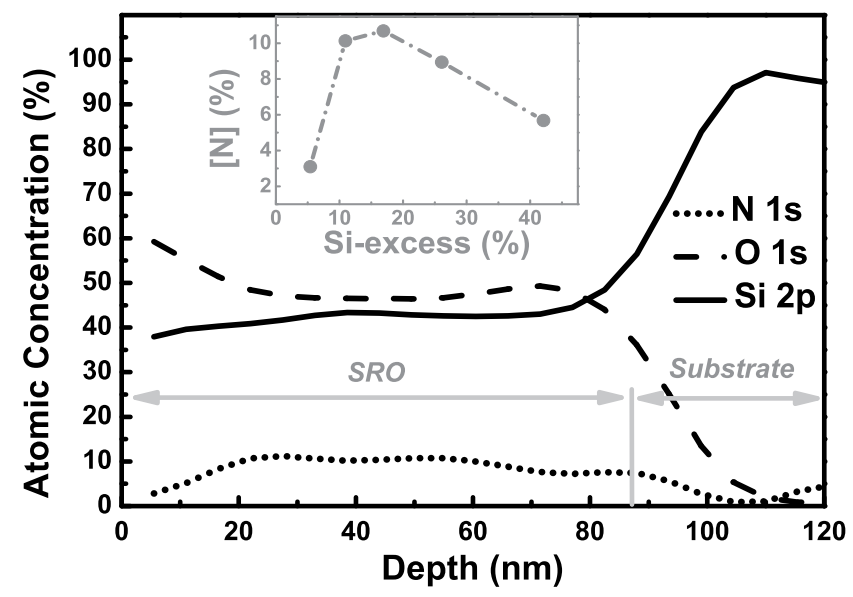

Figure 1. XPS profile of sample P2. The inset shows the nitrogen impurities in the active layer as a function of the $\mathrm{Si}$ excess

The hydrogen comes from the decomposition of silane $\left(\mathrm{SiH}_{4}\right)$ gas precursor, so the densification of the layers is more evident in samples with high Si contents. Figure 1 shows the XPS profile of sample P2 (10.9\% of Si excess) as representative of the whole set. At the surface, the high concentration of oxygen is a clear evidence of oxidation by air exposure. The silicon excess profile is quite flat in the oxide region, a desirable feature for the achievement of uniform Si-nc distributions. It is worth noting the relatively high concentration of $\mathrm{N}$ atoms observed in all the samples (about $10 \%$ in sample P2) that are associated to the $\mathrm{N}_{2} \mathrm{O}$ precursor. The inset of figure 1 represents the nitrogen content as a function of the silicon excess. For the sample close to the stoichiometry (sample P1 with only $5.4 \%$ of Si excess), the relatively ordered matrix hinders the incorporation of impurities, resulting in a low $\mathrm{N}$ concentration. As the $\mathrm{Si}$ excess increases, the nitrogen content reaches its maximum value (sample P2, 10.9\% of Si excess) favored by the increasing disorder of the matrix. A further increase of the $\mathrm{Si}$ content leads to the reduction of the $\mathrm{N}$ concentration, since the low $\mathrm{N}_{2} \mathrm{O} / \mathrm{SiH}_{4}$ ratio reduces the probability of $\mathrm{N}$ incorporation. Figure 2(a) shows the FTIR spectra of layer P2 before and after annealing as an example of PECVD samples. The as-deposited asymmetric Si-O stretching mode ( $\mathrm{TO}_{3}$ mode) exhibits a red-shift of more than $50 \mathrm{~cm}^{-1}$ (peaking at $1020 \mathrm{~cm}^{-1}$ ) with respect to its typical position in pure $\mathrm{SiO}_{2}$ (placed in the range $\sim 1070-1080 \mathrm{~cm}^{-1}$ [18]), which is a clear signature of the non-stoichiometry and matrix disorder. The band observed at $866 \mathrm{~cm}^{-1}$ is probably due to the combination of the asymmetric Si-N stretching mode at $\sim 830-840 \mathrm{~cm}^{-1}$, and the vibrations of two polysilane $\left(\mathrm{SiH}_{2}\right)_{n}$ units, typically observed at $890 \mathrm{~cm}^{-1}$ and $845 \mathrm{~cm}^{-1}$, respectively[19]. After high temperature annealing, the Si-O $\mathrm{TO}_{3}$ mode shifts up to $1065 \mathrm{~cm}^{-1}$, which is a sign of silicon precipitation and phase separation. The attenuation of the $866 \mathrm{~cm}^{-1}$ band is due to the out-diffusion of hydrogen atoms, as deduced from the layer compaction reported from ellipsometry measurements (see table 1 ). In order to characterize the Si-nc distribution profile of PECVD samples, cross section images of sample P2 were 

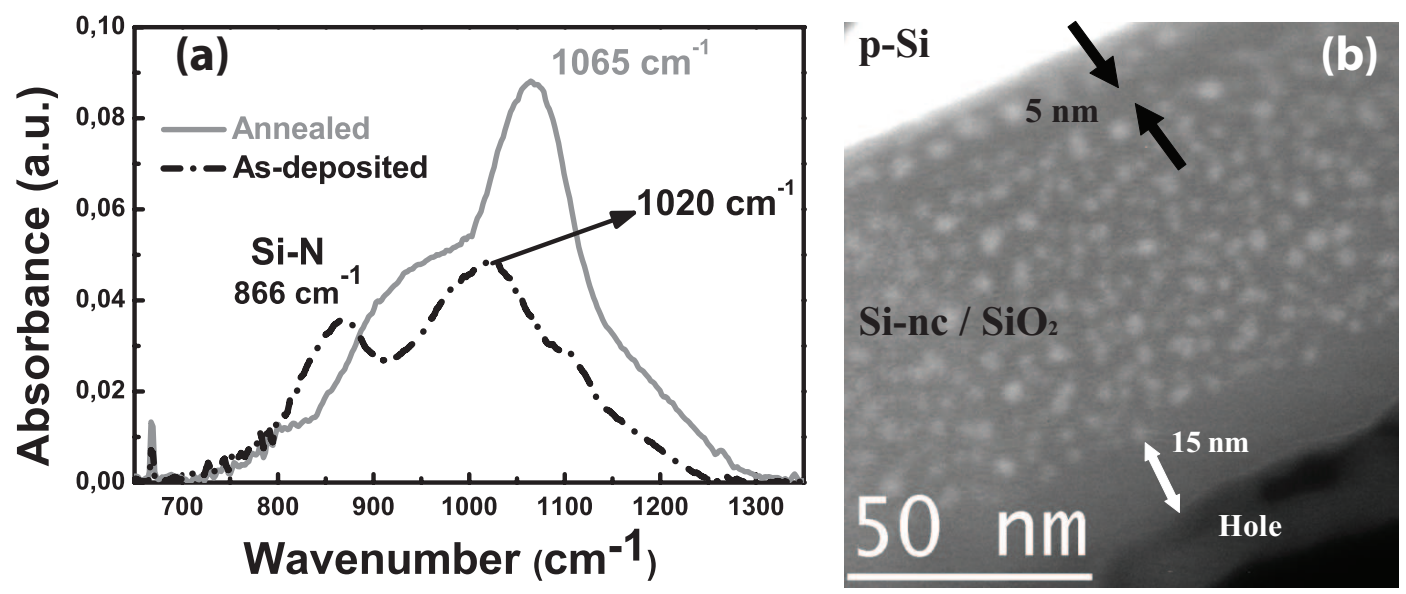

Figure 2. (a) Comparison of FTIR spectra of sample P2 as-deposited and after annealing. (b) EFTEM profile image of the same sample after annealing where a thin depletion of Si-nc distri- bution is observed close to the substrate.

performed by EFTEM [see figure 2(b)]. In good agreement with the flat Si profile observed by XPS, the Si-nc distribution is quite uniform, with a mean size of $\sim 3.60 \mathrm{~nm}$, (standard deviation of $\sigma= \pm 0.50 \mathrm{~nm}$ ), a density of nanocrystals of about $9.0 \times 10^{17} \mathrm{~cm}^{-3}$ and an average distance between closest nanocrystals of about $4 \mathrm{~nm}$. An important result depicted in figure $2(\mathrm{~b})$ is the appearance of a $\sim 5 \mathrm{~nm}$ Si-nc-free region close to the substrate, which has an important impact on PECVD devices (as it will be discussed hereafter). This absence of nanoparticles is attributed either to a diffusion of Si atoms to the substrate during the annealing and to the characteristic deposition instabilities at the first stages of the PECVD process. On the other hand, it is worth noting the strong oxidation of the surface $(\sim 15 \mathrm{~nm})$, also visible on XPS profiles, induced by the high porosity of the as-deposited layers.

\subsection{Ion Implantation}

Implanted samples were characterized in a similar way. The thickness and the $\mathrm{Si}$ excess of the different layers are indicated in table 2. The thickness was determined by ellipsometry and the content of Si was extracted from the XPS profiles. Figure 3 (a) depicts the XPS profile of sample I3 (11.2\% of Si excess) before the etching of the nitride buffer used during ion implantation. Similarly to PECVD samples, a flat profile of silicon is observed in the active layer in agreement with SRIM simulations. Figure 3(b) shows an example of these calculations that corresponds to sample I3. The XPS profile [figure 3(a)] also reveals a sizeable concentration of nitrogen in the SRO layer, comparable to that observed in PECVD samples. This concentration is closely related to the implantation dose, as shown in the inset of figure 3(a). A qualitative evaluation of the damage in the different samples was performed by FTIR. Figure 4(a) shows the infrared spectrum of sample I3 after annealing (dashed line). The deformation of the Si-N $\left(825 \mathrm{~cm}^{-1}\right)$ and $\mathrm{Si}-\mathrm{O}\left(1070 \mathrm{~cm}^{-1}\right)$ stretching peaks with regard to those from a 

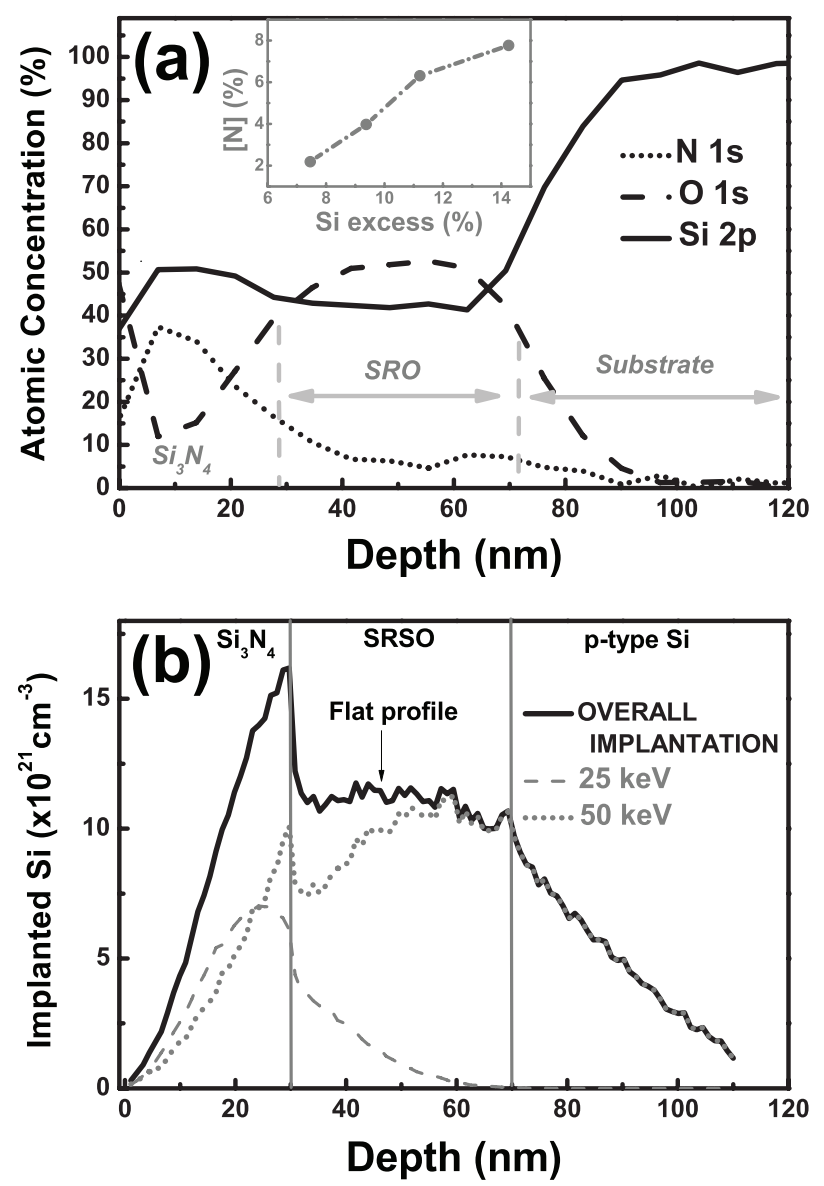

Figure 3. (a) XPS profile of sample I3 acquired before nitride mask etching. The inset shows the variation of the $\mathrm{N}$ content with the implantation dose.(b) SRIM simulation of the implantation profile of sample I3.

non-implanted nitride/oxide stack evidences the effects of the ion implantation. The comparison of the spectra from different samples reveals an increasing deformation of the spectral shape with the silicon concentration (not shown). Figure 4(a) also shows the infrared spectrum of the sample I3 after etching. The total elimination of the Si-N stretching band is noteworthy and underlines the good quality of the chemical etching. EFTEM images were taken in order to study the characteristics of the Si-nc distribution over the $\mathrm{SiO}_{2}$ and $\mathrm{Si}_{3} \mathrm{~N}_{4}$ layers. Figure 4(b) depicts the superposition of the oxygen and nitrogen distributions of sample I3 before nitride etching. The image shows a diffuse $\mathrm{SiO}_{2} / \mathrm{Si}_{3} \mathrm{~N}_{4}$ interface that agrees with the strong diffusion of nitrogen observed in the XPS profiles. The $\sim 5-\mathrm{nm} \mathrm{SiO}_{2}$ layer observed at the surface is a signature of oxidation by air exposure, probably induced by the implantation process. Figure 4(c) shows the Si distribution of the same sample where the Si-nc are clearly observed. The nanocrystals' distribution is continuous and uniform, unlike in PECVD samples where a thin depletion of Si-nc was observed. An average size of $\sim 3.10 \mathrm{~nm}$ (standard deviation $\sigma= \pm 0.47 \mathrm{~nm}$ ) was measured with a Si-nc density of about $1.6 \times 10^{18} \mathrm{~cm}^{-3}$ and an average distance 

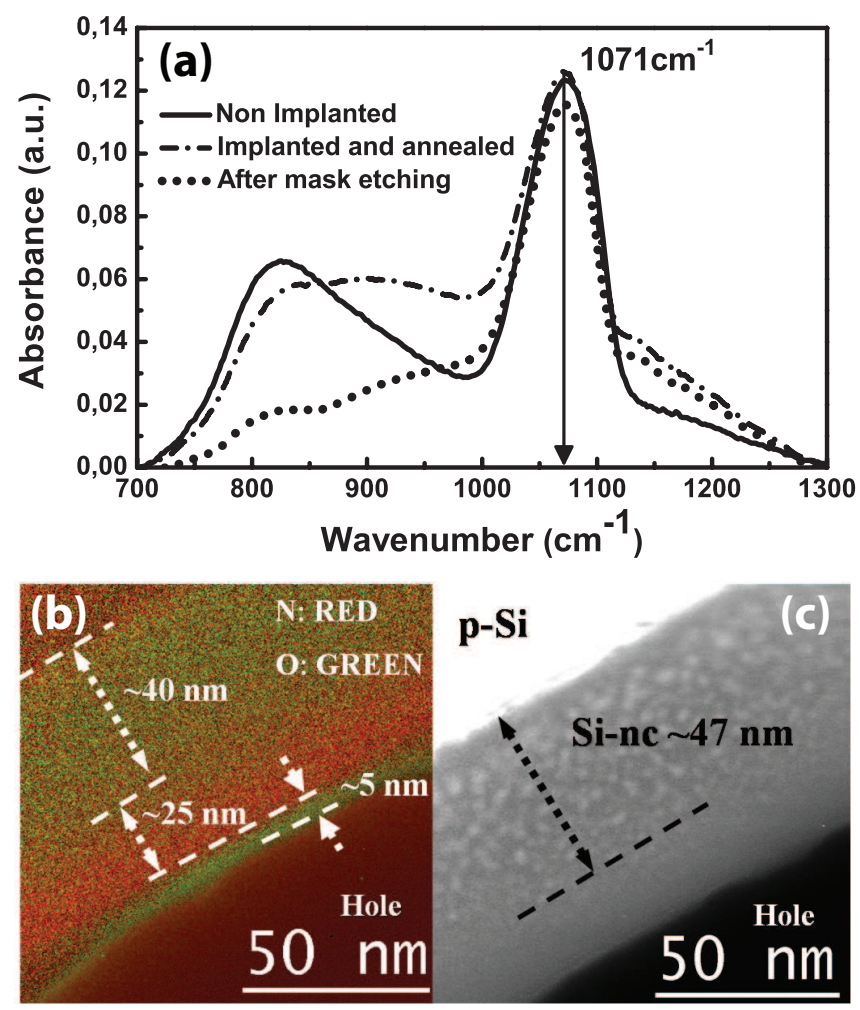

Figure 4. (a) Comparison of FTIR spectra of sample I3 before and after the chemical etching of the nitride layer. At the same time, these spectra are compared with a non implanted $\mathrm{Si}_{3} \mathrm{~N}_{4} / \mathrm{SiO}_{2}$ spectrum. (b) Superposition of the $\mathrm{O}$ and $\mathrm{N}$ distributions of sample I3 measured by EFTEM before the elimination of the mask. (c) Distribution of the Si atoms in sample I3 also measured by EFTEM. The Si-nc are clearly observed.

between closest neighbours of $\sim 3 \mathrm{~nm}$. Note that, in spite of the similar Si contents in samples I3 and P2, the mean Si-nc size is slightly different $(\sim 3.10 \mathrm{~nm}$ in sample I3 as opposed to $\sim 3.60 \mathrm{~nm}$ in sample P2) which is, in some way, compensated by the different Si-nc densities observed. Finally, the comparison of figures 4(b) and 4(c) reveals that the Si-nc distribution partially takes in the nitride layer $(\sim 7 \mathrm{~nm})$ and appears to be linked to an additional PL band, as will be shown later on.

\section{Emission properties}

Figure 5 shows the PL spectra of annealed PECVD samples that exhibit the typical emission in $\mathrm{Si}-\mathrm{nc} / \mathrm{SiO}_{2}$ systems in the 1.4 - $1.7 \mathrm{eV}$ spectral range. The $\mathrm{PL}$ is maximum in sample P2 (10.9\%) whereas in samples with extreme Si contents (i.e. 5.4\%, sample P1 and 42.1\%, sample P5) no emission is observed. The inefficient excitation observed at low Si concentrations is related to the small size (small density of states) and number of grown nanocrystals. In contrast, the poor emission observed for high Si contents is due to the loss of quantum confinement and the apparition of quenching effects with neighbouring nanocrystals [20,21]. Additional PL measurements were performed in the 


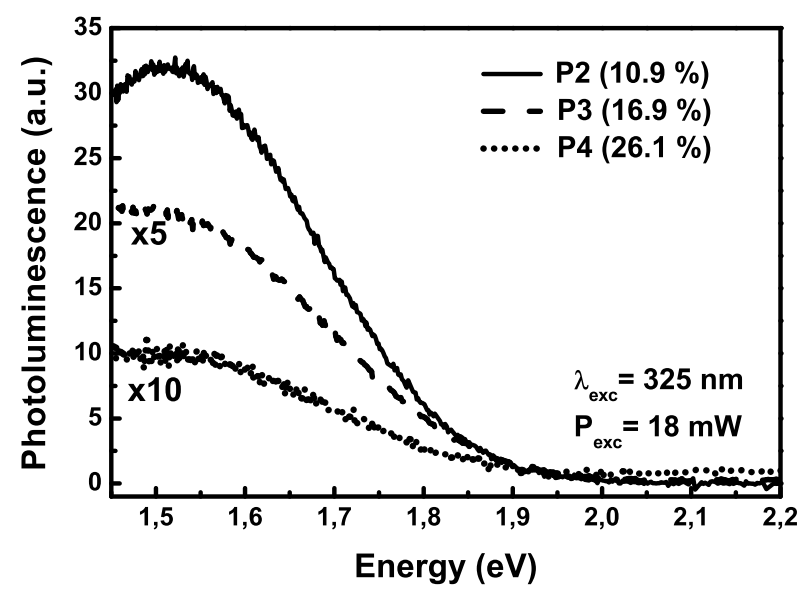

Figure 5. Photoluminescence spectra of different PECVD samples.

NIR range for the whole PECVD set of samples (results not shown) revealing strong band-to-band emission from the Si-substrate at about $1.1 \mathrm{eV}$.
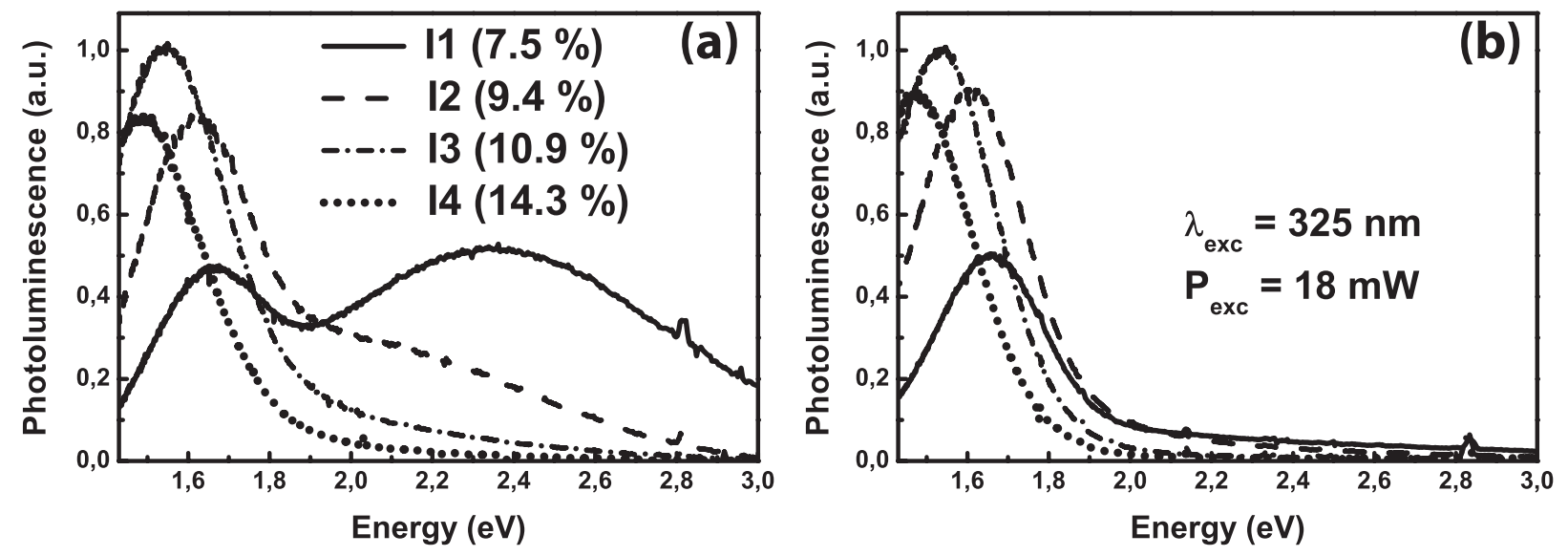

Figure 6. (a) Photoluminescence spectra of implanted samples before nitride etching. (b) The same spectra were measured after nitride removing. (Each spectrum has been normalized to its corresponding layer thickness)

Figure 6(a) shows the emission of the different implanted layers after annealing and before nitride etching. All the samples exhibit two broad emission bands. The first one is placed in the 1.4-1.7 eV spectral range which corresponds to the typical emission from $\mathrm{Si}-\mathrm{nc} / \mathrm{SiO}_{2}$ systems. The second contribution is especially visible in sample I1, and peaks close to $2.4 \mathrm{eV}$. Figure $6(\mathrm{~b})$ represents the PL spectra of the same samples after nitride buffer removing. The band at the high energy range has been completely suppressed, clearly placing its origin in the nitride layer. We have recently proposed the possibility of broad range tuning of the emission by accurately monitoring the thickness ratio of silicon-rich nitride/SRO stacks[22]. The emission mechanisms involved in SRN (silicon-rich nitride) layers still remain unclear, even though the apparent red-shift with 
the Si content could be related to Si-nc precipitates, as in the case of SRO matrices. The PL measurements in the NIR range (not shown) contrast with the results obtained in PECVD samples, since no emission from bulk silicon was observed. This is explained in terms of the deep implantation tails into the Si substrate predicted by SRIM simulations [see figure 3(b)] that damages the local structure, suppressing the typical emission at $1.1 \mathrm{eV}$.
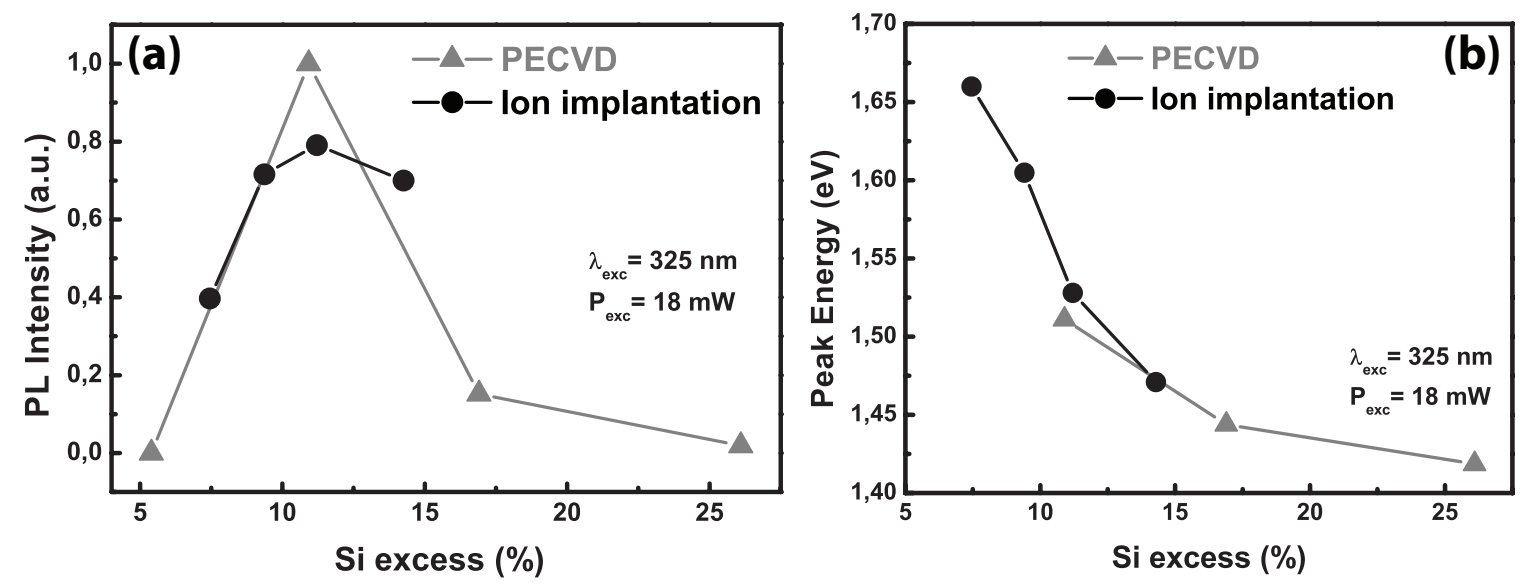

Figure 7. Photoluminescence intensity (a) and peak position (b) as a function of the silicon content.

Figure 7(a) shows the dependence of the photoluminescence intensity in deposited and implanted samples as a function of the Si excess. All the values were normalized to their respective layer thickness and to the most intense emission value obtained from sample P2. The plot reveals a good agreement between both PECVD and implanted samples with a maximum emission intensity found for layers with $\sim 11 \%$ of Si excess, samples I3 and P2. Taking into account the density of Si-nc calculated from EFTEM images, one can expect that sample I3 would exhibit a higher PL intensity. However, the emission from sample I3 is approximately $20 \%$ lower than that of sample P2, which probably suggests that the increase of the density of Si-nc is detrimental, as the interaction between neighbours and non-radiative processes are favoured, in spite of the smaller size of the Si-nc ( $\sim 3.1 \mathrm{~nm}$ as opposed to $\sim 3.6 \mathrm{~nm}$ in sample P2). Figure 7(b) depicts the variation of the emission energy as a function of the Si excess for implanted and deposited structures. An overall correlation of the behaviour regarding the $\mathrm{Si}$ content was observed in all the samples independently of the elaboration technique. Indeed, the enlargement of the Si-nc size (i.e. the Si excess) induces a decrease in the band gap, leading to the observed red-shift of the emission[23, 24, 25]. Samples P2 and I3, with similar Si excesses (10.92\% and $11.21 \%$, respectively), exhibit slightly different emission energies, which is attributed to the different sizes as revealed by EFTEM images. Figures 8(a) and 8(b) represent the PL spectra of samples P2 and I3 as a function of the incident photon flux. The measurements were performed by pumping with an Argon laser operating at $488 \mathrm{~nm}$. On the represented 3D surface plots, the 

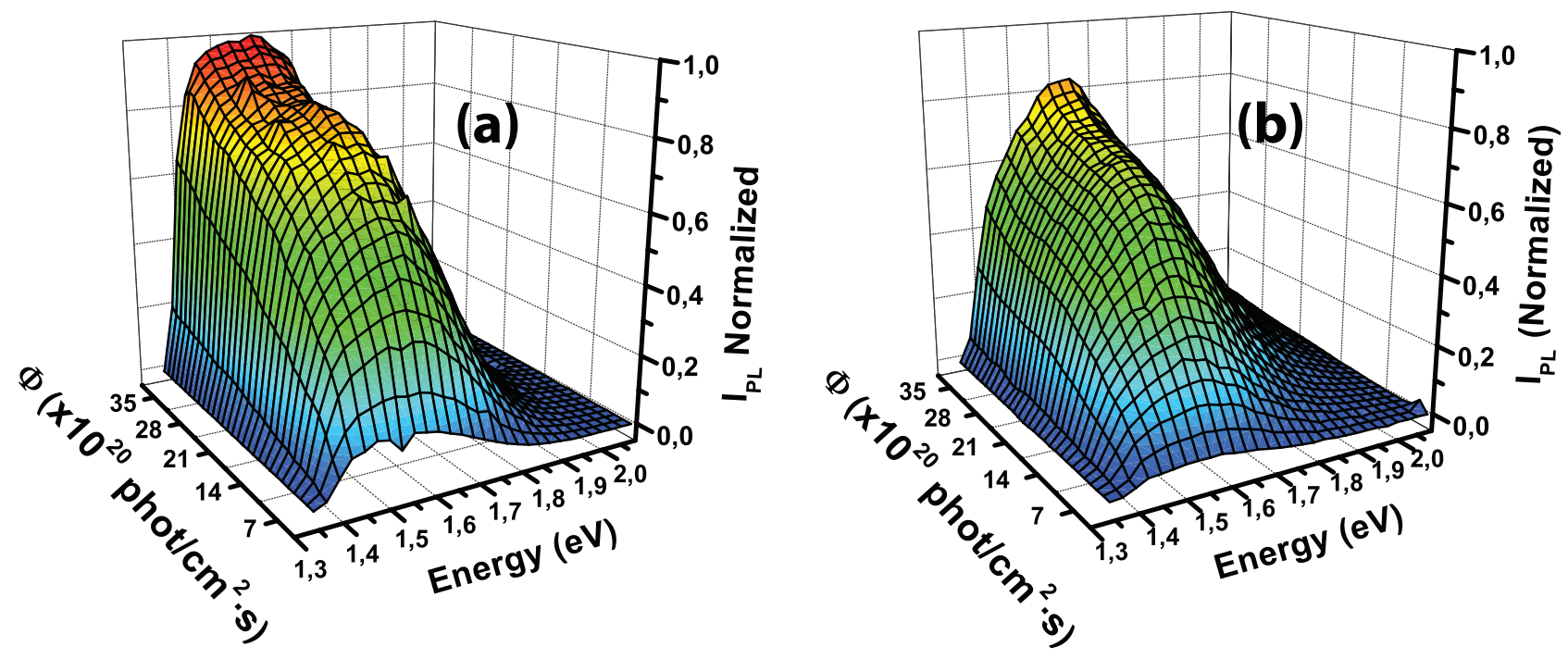

Figure 8. PL spectra of samples P2 (a) and I3 after chemical etching (b) as a function of the incident photon flux.

grid lines parallel to the energy axis indicates the spectra at different excitation fluxes, whereas the grid lines parallel to the photon flux axis represents the PL saturation curves at different detection wavelengths. The intensity of the PL was normalized, in both samples, to their respective thickness and to the most intense emission, that is, to the emission from sample P2. Due to the fast generation of excitons (as will be shown later), the PL intensity can be described, in first approximation, by means of a quasi-two level system. This simple model only considers two energy levels, corresponding to the top of the valence band (fundamental state $A$ ) and the bottom of the conduction band (excited state $B$ ). The rate equations can be expressed by:

$$
\begin{aligned}
& \frac{d N_{B}}{d t}=-\frac{N_{B}}{\tau_{R A D}}-\frac{N_{B}}{\tau_{n R A D}}+N_{A} \cdot \sigma_{a b s} \cdot \Phi \\
& \frac{d N_{A}}{d t}=-\frac{d N_{B}}{d t} \\
& N_{A}+N_{B}=N_{n c}=\text { const. }
\end{aligned}
$$

where $N_{A}$ and $N_{B}$ are the populations of the levels $A$ and $B, \tau_{R A D}$ and $\tau_{n R A D}$ are the radiative and non-radiative decay times, respectively, $\Phi$ is the incident photon flux and $\sigma_{a b s}$ is the absorption cross-section of the Si-nc at the excitation wavelength. $N_{n c}$ is the number of Si-nc. Note that, in these equations, we are not taking into account any second order effects, such as two photon absorption and Auger processes.

In the steady state: $d N_{B} / d t=0$, and the population of the excited state is given by:

$$
N_{B}=N_{n c} \cdot \frac{\sigma_{a b s} \cdot \Phi}{\sigma_{a b s} \cdot \Phi+\tau_{P L}^{-1}}=N_{n c} \cdot \frac{\Phi}{\Phi+\frac{1}{\sigma_{a b s} \cdot \tau_{P L}}}
$$

with $\frac{1}{\tau_{P L}}=\frac{1}{\tau_{R A D}}+\frac{1}{\tau_{n R A D}}$. 
The measured PL intensity, $I_{P L}$, is proportional to the emitted photon flux $\Phi_{e m}=N_{B} / \tau_{R A D}$ and depends on both the excitation and detection energy, $E_{\text {exc }}$ and $E$ respectively. Therefore, $I_{P L}$ can be written in the form,

$$
I_{P L}\left(E_{e x c}, E\right) \propto \frac{1}{\tau_{R A D}(E)} \cdot N_{n c}(E) \cdot \frac{\Phi}{\Phi+\frac{1}{\sigma_{a b s}\left(E_{e x c}, E\right) \cdot \tau_{P L}(E)}}
$$

where the dependence of the different parameters with the excitation and the detection energies is explicitly expressed. According to this equation, the intensity of PL as a function of the incident photon flux is linear for low flux values and saturates for very high excitation powers. Moreover, equation 5 shows that the term $N_{n c} / \tau_{R A D}$ gives the maximum intensity achievable, while the product $\sigma_{a b s} \cdot \tau_{P L}$ marks the flux value of saturation, so that the higher is the value of this product the lower is the saturation flux. In sample P2 [figure $8(\mathrm{a})$ ], at $\sim 1.52 \mathrm{eV}$ (approximately the peak energy), the linear region of the saturation curve takes place in the range from zero to $5 \times 10^{20}$ photons $/\left(\mathrm{cm}^{2} \cdot \mathrm{s}\right)$ and the saturation is achieved for fluxes of about $2 \times 10^{21}$ photons $/\left(\mathrm{cm}^{2} \cdot \mathrm{s}\right)$. In contrast, for sample I3 [figure 8b] at the same detection energy, the linear region is slightly larger, up to $7.5 \times 10^{20}$ photons $/\left(\mathrm{cm}^{2} \cdot \mathrm{s}\right)$, and the saturation regime is reached at $2.5 \times 10^{21}$ photons $/\left(\mathrm{cm}^{2} \cdot \mathrm{s}\right)$. It should be noted that these behaviours are in agreement with the respective values of $\sigma_{a b s} \cdot \tau_{P L}$, as extracted from figures 10 and 11 (presented below). Figures 8(a) and 8(b) also show how, after the PL saturation occurs, a further increase of the excitation power (above $3 \times 10^{21}$ photons $/\left(\mathrm{cm}^{2} \cdot \mathrm{s}\right.$ ) generates an additional increase of the emission intensity. A similar effect is observed in 1D structures fabricated by reactive magnetron sputtering[26]. This behaviour is attributed to the increase of Si-substrate band-to-band transitions that, for very high incident powers can partially take in the visible range. This effect is especially seen in sample P2, which is compatible with the strong emission observed at around $1.1 \mathrm{eV}$ in the NIR range spectra of PECVD samples.

The increase of the pumping power also affects the energy of emission [see figure $9(\mathrm{a})$. In a Si-nc distribution, larger nanocrystals are easily excited thanks to their longer lifetimes and to the energy transfer from smaller quantum dots[21]. As the incident photon fluxes increase, the probability to excite smaller nanocrystals increases while the emission of the large Si-nc saturates. Consequently, the PL emission shifts to shorter wavelengths (higher energies), as depicted in figure 9(a). In a similar way, if the excitation energy is increased, smaller Si-nc can be easily excited shifting the emission towards the blue spectral range and increasing the overall PL intensity, as can be seen in figures $9(\mathrm{~b})$ and $9(\mathrm{c})$.

In order to calculate the absorption cross-section of the different samples (implanted and PECVD), time-resolved measurements were performed for different detection wavelengths (energies): $700 \mathrm{~nm}(\sim 1.77 \mathrm{eV}), 750 \mathrm{~nm}(\sim 1.65 \mathrm{eV}), 800 \mathrm{~nm}(\sim 1.55 \mathrm{eV})$ and $850 \mathrm{~nm}(\sim 1.46 \mathrm{eV})$. The excitation was performed with an Argon laser operating at 457 $\mathrm{nm}(\sim 2.71 \mathrm{eV})$ and $488 \mathrm{~nm}(\sim 2.54 \mathrm{eV})$, even though in the former case only low-flux pumping was applied. From the rate equations presented above, we can describe the 

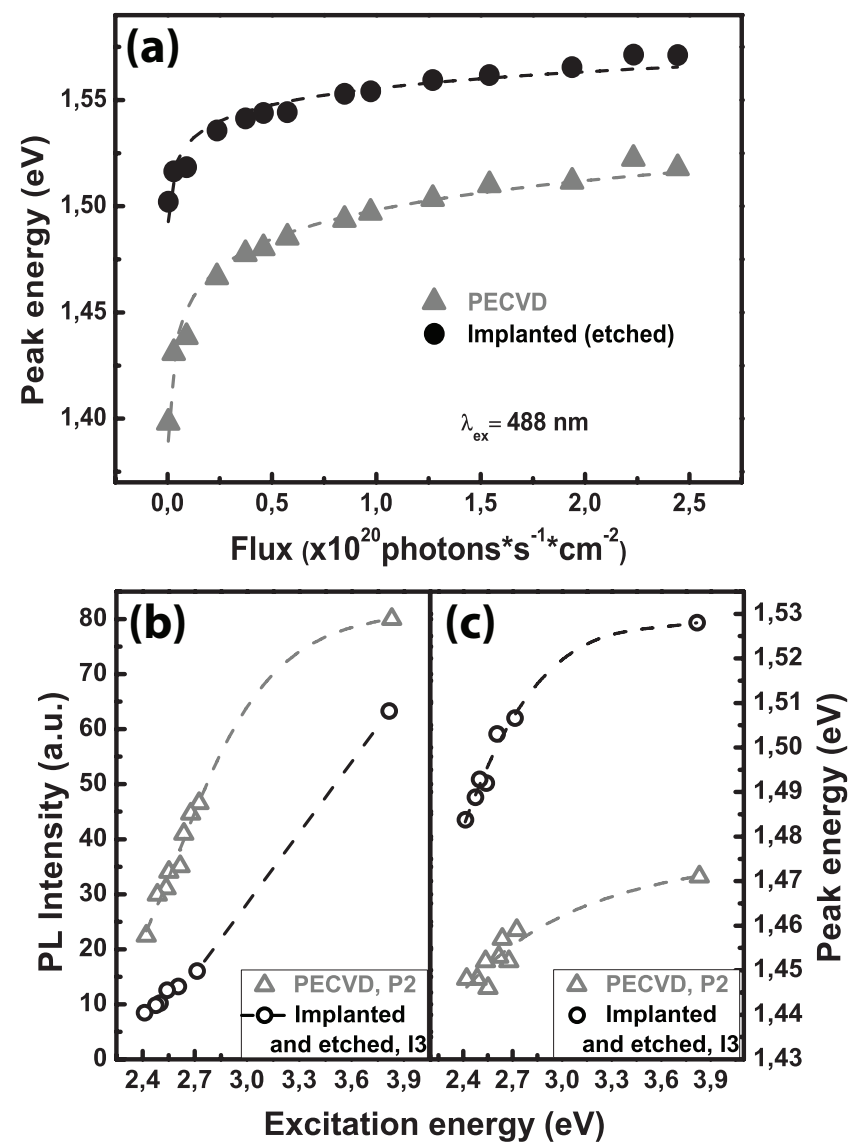

Figure 9. (a) Variation of the PL peak energy of samples I3 and P2 as a function of the pumping power. PL intensity (b) and peak energy (c) dependence with the excitation energy.

transient behaviour of $\mathrm{I}_{P L}$ when the pump is switched on (at $t=t_{0}$ ) by simply integrating $d N_{B} / d t$. Therefore, $\mathrm{I}_{P L}$ reads,

$$
I_{P L}(t)=I_{P L}^{\max } \cdot\left[1-\exp \left(-\frac{t-t_{0}}{\tau_{\text {rise }}}\right)\right]
$$

where $\mathrm{I}_{P L}^{\max }$ is the maximum of the PL intensity (at the stationary state) and $\tau_{\text {rise }}$ is given by,

$$
\frac{1}{\tau_{\text {rise }}}=\frac{1}{\tau_{P L}}+\sigma_{a b s} \cdot \Phi
$$

From this relation it is possible to calculate the value of the absorption cross-section, $\sigma_{a b s}$, at different pumping powers by directly obtaining $\tau_{\text {rise }} \tau_{P L}$ from the rise and decay edges of the emission. Experimentally, it has been observed that the transients are best described by stretched exponential functions,

$$
I_{P L}(t)=I_{P L}^{\max } \cdot \exp \left(-\left[\frac{t}{\tau_{P L}}\right]^{\beta}\right)
$$

where the exponent $\beta$ gives an idea of the lifetime dispersion in a Si-nc distribution [27]. Figures 10(a) and 10(b) show the decay times as a function of the detection energy for 
PECVD and implanted samples, respectively. In both cases, the PL lifetimes decrease with the emission energy, that is, with decreasing Si-nc size. This is explained by the loss of quantum confinement in larger nanocrystals with the consequent decrease of the radiative rate. The similarity of the emission lifetimes under 488-nm and 457-nm

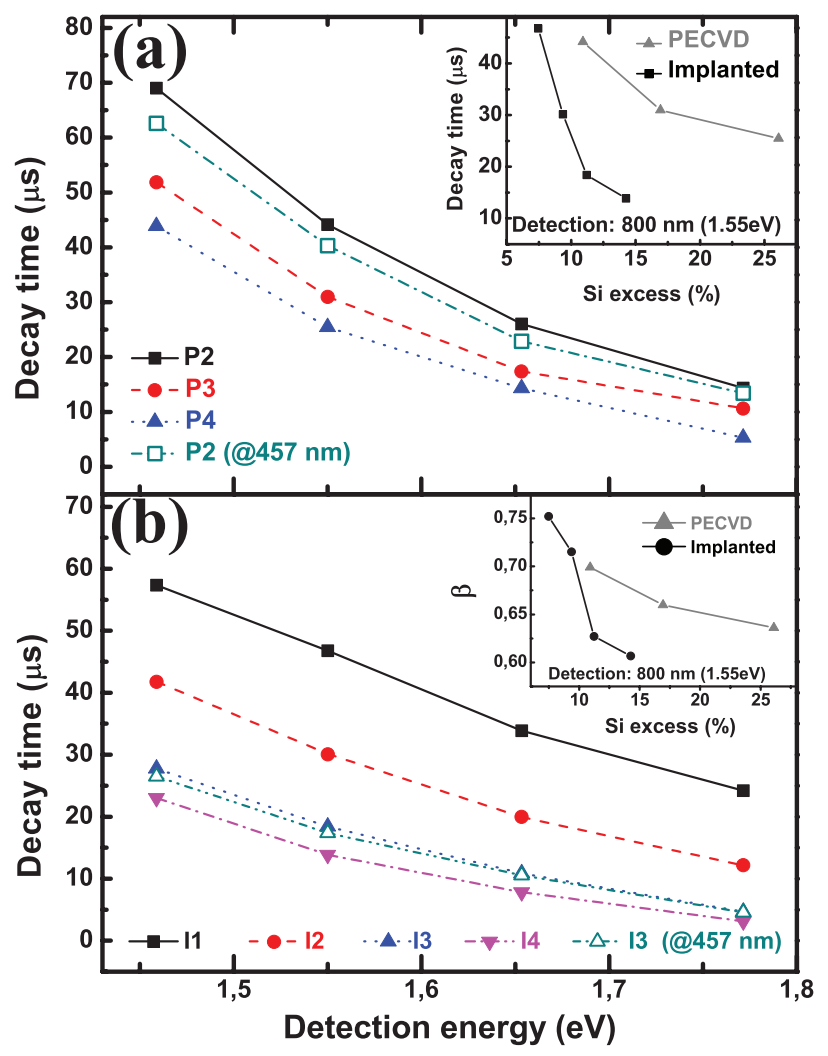

Figure 10. (a) Emission decay times of PECVD samples under 488-nm excitation obtained from stretched exponential fits. Also decay times of sample P2 under 457$\mathrm{nm}$ pumping are represented. (b) Decay times of implanted samples under identical conditions. In the insets the decay times and $\beta$ parameters of both kinds of samples are represented as functions of the Si content at a detection wavelength of $800 \mathrm{~nm}$.

excitations in samples P2 and I3 indicates that the initial relaxation of the Si-nc to the emission level is much faster than typical Si-nc emission lifetimes. The inset of figure 10(a) represents the decay times of PECVD and implanted samples as a function of the Si-excess, at a detection wavelength of $800 \mathrm{~nm}$. As the $\mathrm{Si}$ excess increases, the larger size of the Si-nc and the higher number of defects in the surrounding matrix favour the non-radiative relaxations, leading to shorter emission lifetimes. A faster decrease is observed in implanted samples that is probably due to the higher density of Si-nc and the shorter interdot distance observed by EFTEM. In the same way, a fast decrease of $\beta$ parameter at $800 \mathrm{~nm}$ was found in the implanted samples that contrasts with a relatively slow variation in the PECVD samples [see inset of figure 10(b)]. The $\beta$ parameter varies from 0.60 to 0.75 in agreement with typical values reported in the literature[27].

The absorption cross section of PECVD and implanted samples can be calculated 

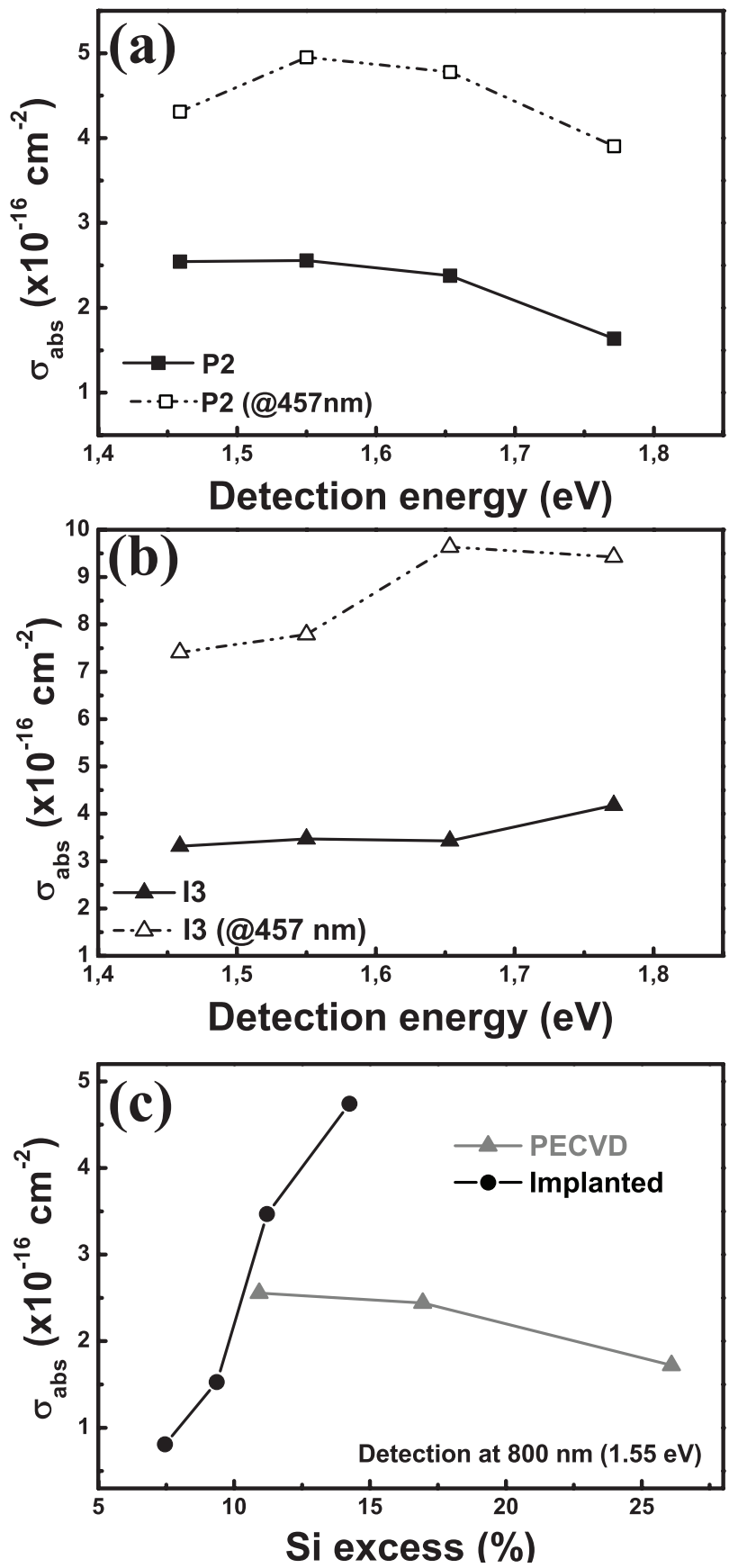

Figure 11. Absorption cross-sections of sample P2 (a) and I3 (b), in both cases obtained under 488-nm and 457-nm excitation. (c) Dependence of $\sigma_{a b s}$ with the $\mathrm{Si}$ content for both sets. 
from equation 7 , from the rise and decay times of the time resolved PL measurements. Figures 11(a) and 11(b) show the values of $\sigma_{a b s}$ for samples P2 and I3, respectively at the excitation wavelengths of $488 \mathrm{~nm}$ and $457 \mathrm{~nm}$. In sample P2 [figure 11(a)], $\sigma_{a b s}$ decreases with the detection energy due to the reduction of the density of absorbing states in smaller nanocrystals, in good agreement with results reported by Kovalev et al.[28]. In implanted samples the behaviour is quite different, since a slight increase of $\sigma_{a b s}$ with the detection energy is observed. The same behaviour has been reported in similar structures in reference[29]. In that work, C. Garcia et al. attributed this variation to an increase of the oscillator strength for smaller nanocrystals that compensates the reduction of the density of states. Figure 11(c) shows the dependence of the absorption cross section with the Si excess of PECVD and implanted samples at a detection wavelength of 800 $\mathrm{nm}(1.55 \mathrm{eV})$. In implanted samples, $\sigma_{a b s}$ rapidly increases with the nanocrystal size ( $\mathrm{Si}$ excess) as more states are accessible to the 488-nm excitation. This behaviour contrasts with that observed in PECVD samples, where a slight decrease of $\sigma_{a b s}$ is observed with the Si excess, probably due to the presence of a higher number of non-radiative recombinations (higher number of defects).

In addition it is worth noting the increase of the absorption cross-section of samples I3 and P2 when the pumping wavelength is switched from $457 \mathrm{~nm}(\sim 2.71 \mathrm{eV})$ to 488 $\mathrm{nm}(\sim 2.54 \mathrm{eV})$. The increase of the pumping energy increases the number of accessible states and leads to an enhancement of the absorption cross-section.

\section{Device characterization}

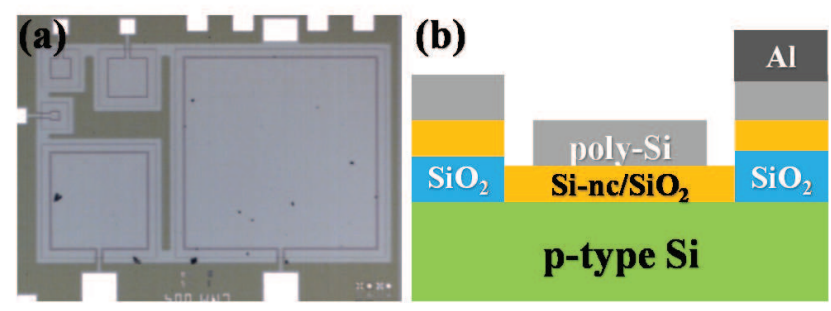

Figure 12. (a) Top view image of fabricated MOS devices with different contact areas. (b) Cross section view of the MOS structures.

Metal-oxide-semiconductor structures with different active areas were fabricated by means of a standard photolithographic process. Figure 12(a) is a top-view image of the device. A basic cross-section scheme of these devices can be seen in figure 12(b). The top-electrode is a 350-nm semitransparent poly-Si with an average transmittance of about $50 \%$ in the range of interest. The active layers typically have $\sim 45-50 \mathrm{~nm}$ and have been prepared with different Si contents, taking into account the results depicted in figure $7(\mathrm{a})$. In order to maximize the signal-to-noise ratio of the electroluminescence trace, this work is mainly focused on devices with larger electrode areas $\left(9.6 \times 10^{-3} \mathrm{~cm}^{2}\right.$ and $\left.2.3 \times 10^{-3} \mathrm{~cm}^{2}\right)$. 


\subsection{PECVD}

Table 3 summarizes some basic characteristics of MOS structures fabricated by PECVD. The Si contents were estimated from the nominal $\left[\mathrm{N}_{2} \mathrm{O}\right] /\left[\mathrm{SiH}_{4}\right]$ ratios, considering the experimental values obtained for PECVD test layers (shown in table 1) and the thickness was obtained by ellipsometry. The slight increase of the thickness is associated with the addition of $\mathrm{Si}$, since all the layers were fabricated with identical deposition times.

In order to characterize the dielectric permittivity, $\epsilon_{r}$, of the different samples and to calculate the density of trapped charge inside the SRO, $N_{t}$, forward-backward C$\mathrm{V}$ characteristics were measured [see figures 13(a) and 13(b)]. To assure an effective carrier injection, the voltage sweeps were adjusted for each sample taking into account its respective onset of emission and breakdown voltage (if any). The parameter labeled as $C_{\max }$ in table 3 represents the maximum capacitance of the structure (capacitance in accumulation regime) and is directly related with the relative permittivity of the layer, $\epsilon_{r}$, by the following equation,

$$
C_{\max }=\frac{\epsilon_{r} \epsilon_{0} S}{d}
$$

where $\epsilon_{0}$ is the vacuum permittivity, $S$ is the area of the device and $d$ is the thickness of the active layer. In the inset of figure 13(a), $\epsilon_{r}$ is represented as a function of the silicon excess. As expected, the effective dielectric permittivity increases due to the progressive incorporation of $\mathrm{Si}$ into the active layer. The trapped charge is calculated from the shifts of the flat-band voltage, $\Delta V_{F B}$, measured from a reference $\mathrm{C}-\mathrm{V}$ curve of a fresh device,

$$
N_{t}=\frac{C_{\max } \Delta V_{F B}}{e S}
$$

The values of $N_{t}$ are shown in table 3 . A general increase of $N_{t}$ with the Si excess is observed and attributed to the enhanced conductivity of the SRO layers and to the increase of the average Si-nc size that facilitates the carrier trapping. The $\mathrm{C}-\mathrm{V}$ measurements reveal two different behaviours that are distinguished in figures 13(a) and 13(b). The first case, depicted in figure 13(a), corresponds to samples with low Si contents (PD2 and PD3) and is characterized by a small density of trapped charge, $10^{10}-10^{11} \mathrm{~cm}^{-2}$. In these samples, the thin zone depleted of Si-nc strongly limits the carrier injection from the substrate that is particularly observed above $\sim 20 \mathrm{~V}$ when the Fowler-Nordheim mechanism is strong enough to efficiently inject the carriers into the Si-nc, as will be shown later on. As the Si excess further increases (up to $\sim 13.7 \%$, sample PD4), the C-V plots show a significant increase in the number of carriers injected from the substrate (electrons and holes) [see figure 13(b)]. In this case, the higher Si concentration facilitates the tunnelling from the substrate at lower voltages, leading to densities of trapped charge of $\sim 10^{12} \mathrm{~cm}^{-2}$. Note that the variation of the trapped charged with the Si excess (in the presence of the 5-nm oxide free of Si-nc) is very similar to that observed when the tunnel oxide thickness is modified, as it has been reported in reference [30]. 

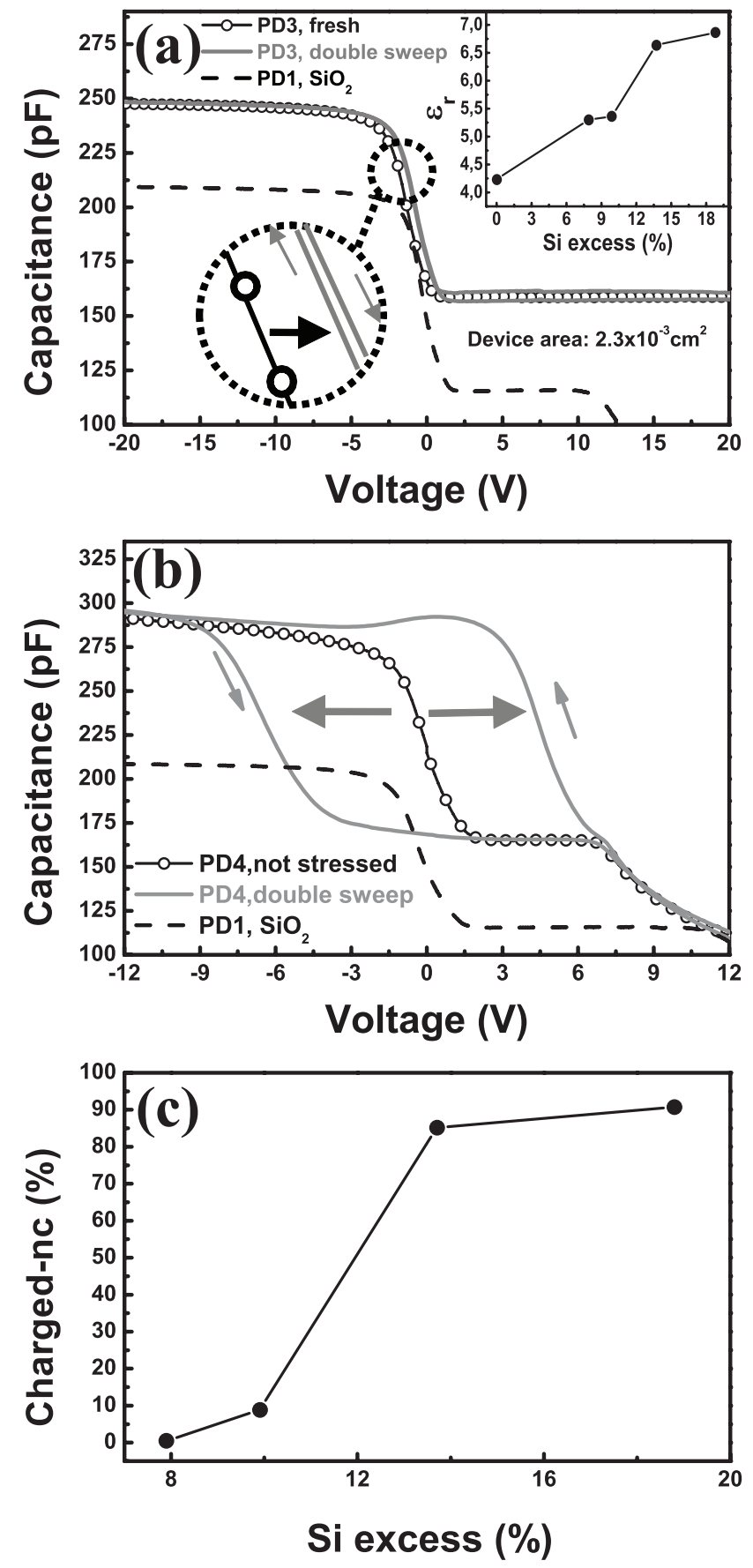

Figure 13. (a) Capacitance-voltage curve of sample PD3. In the inset the dielectric permittivity of the different PECVD samples is represented. (b) Capacitance-voltage curve of sample PD4. The grey arrows indicate the flat-band voltage shift and the black ones indicate the sweep direction. (c) Variation of the ratio of charged-nc as a function of the Si excess. 
The density of trapped charge, in combination with the Si-nc density obtained by EFTEM, leads to a rough estimation of the percentage of charged nanocrystals. This parameter is rather an upper bound to the ratio of excitable Si-nc and, in some way, can be considered as a marker of the room available to further improve the excitation efficiency, since it also indicates the ratio of non-active Si-nc. The calculation is performed under the assumption that most of the trapped charge is associated to the Si-nc, which is justified by the low value of $N_{t}$ observed in pure oxide structures (device PD1). Nevertheless, it is also true that the addition of the Si-excess causes the generation of defects and traps not observed in $\mathrm{SiO}_{2}$ that probably leads to an overestimation of the number of active Si-nc. Figure 13(c) shows the results of these calculations. Devices PD4 and PD5 exhibit the higher occupation ratios with values close to $\sim 90 \%$, whereas in structures PD2 and PD3 the percentage of Si-nc that, a priori, will contribute to the emission is very low, up to $\sim 0.4 \%$ and $\sim 8.9 \%$, respectively. Note that the presence of a large number of trapped charges does not necessarily lead to more intense emission since it reinforces as well the apparition of fast Auger de-excitations. In order to study the conduction mechanisms, quasi-static current-voltage characteristics were acquired under forward-backward voltage sweeps. Figure 14 shows an example of these measurements

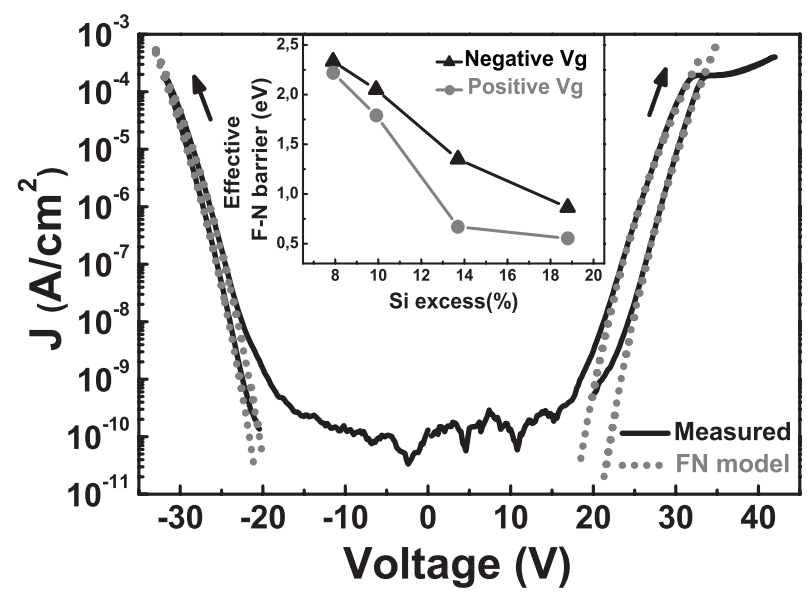

Figure 14. Forward and backward current-voltage characteristics of the structure PD3. The arrow shows the forward sweep direction. The inset depicts the variation of the effective barriers extracted from FN modeling during the forward sweep.

that corresponds to the structure PD3. In all cases, the current, in the range of interest (emission range), is well fitted by the Fowler-Nordheim (FN) tunneling expression. This injection mechanism takes place when the applied gate voltage, $V_{g}$, raises the Fermi level so that carriers see a triangular barrier. Although theoretically, this always occurs when $q V_{g} \Phi_{b}$ (injection barrier height), in practice, FN currents are usually observed at higher fields. The FN current expression reads,

$$
J_{F N}=\frac{q^{2} m_{0}}{8 \pi h m_{o x}^{*} \Phi_{b}} E^{2} \exp \left(-\frac{8 \pi \sqrt{2 q m_{o x}^{*}} \Phi_{b}^{3 / 2}}{3 h E}\right)
$$


where $m_{0}$ is the stationary electron mass, $m_{o x}^{*}$ is the effective mass of carriers in the $\mathrm{SiO}_{2}$ band under consideration, $h$ is the Plancks constant, and $E$ the applied electric field. The onset of the FN injection is strongly dependent on the Si content, varying from 6-7 $\mathrm{V}$ in sample PD5 $(\sim 18.8 \%)$ to $23 \mathrm{~V}$ in sample PD2 $(\sim 7.9 \%)$. As will be seen later, these values are closely related to the obtaining of detectable field-effect EL. From the FN fits, it is possible to extract the value of $\Phi_{b}$. It should be noted that due to the presence of the Si-nc, $\Phi_{b}$ represents the effective height between the quantized states of the silicon nanocrystals and the top of the oxide barrier. The inset of figure 14 shows the barrier height obtained for positive and negative voltage sweeps as a function of the Si excess. Only barriers in the forward sweep direction are represented since they are less affected by the carrier trapped. In the light of the figure, it becomes clear that, independently of the bias polarity, the increase of the Si excess facilitates the carrier transport through the structure, which translates into a barrier height lowering. On the other hand, it is also noteworthy the good agreement between the effective barriers observed for positive and negative voltages and the kind of carriers injected from the substrate. Figure 15
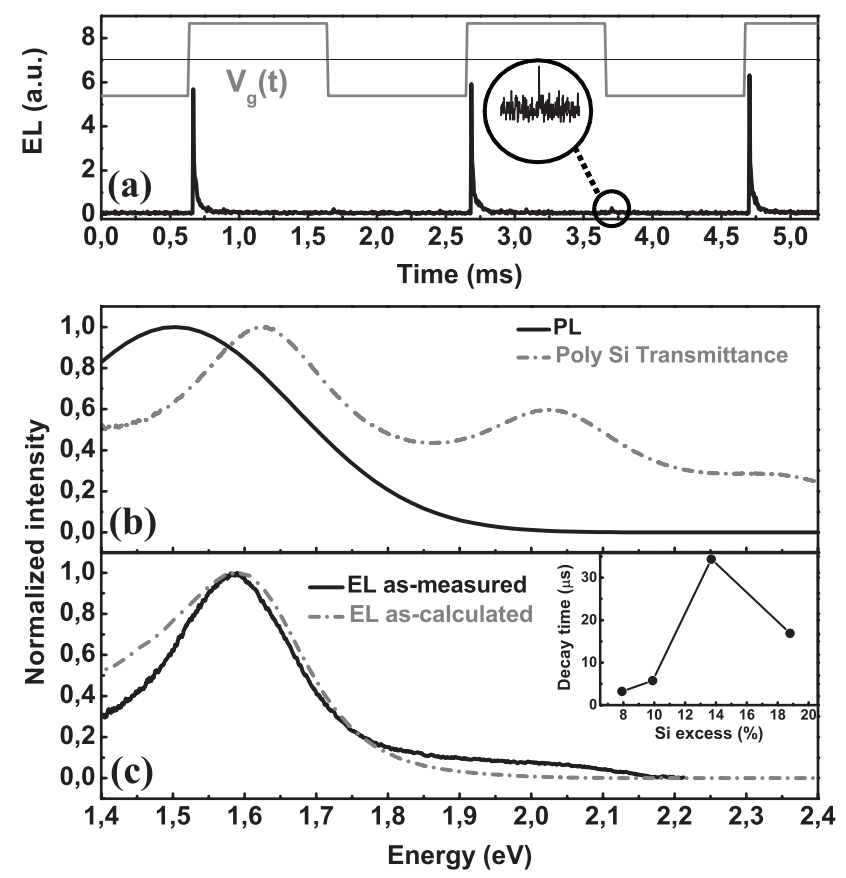

Figure 15. (a) Time-resolved electroluminescence of sample PD3 under $25 \mathrm{~V}$ alternate polarization.(b) The PL spectrum of layer P2 (without top electrode) versus the polytop electrode transmittance. In the inset, the EL decay times of the different samples are represented (c) Com- parison between the as-measured EL spectrum (from device PD3) and that obtained from the combination of the poly-Si transmittance and the PL spectrum given in (b).

summarizes the behaviour of the field-effect luminescence in PECVD structures. Figure 15(a) shows the time-resolved EL of sample PD3 under application a square wave with an amplitude of $25 \mathrm{~V}$, whereas figures 15 (b) and 15(c) represent the PL and EL spectra of similarly prepared samples, respectively. The onset of the emission decreases with 
the Si content from $\sim 20 \mathrm{~V}$, in structure PD2 (7.9\%), to $\sim 6 \mathrm{~V}$, in sample PD5 (18.8\%), as depicted in figure 16(a). It should be noted the good accordance between the onsets of emission and FN injection, which clearly links the field effect emission of PECVD samples with this injection mechanism. The field-effect mechanism entails the sequential injection of electrons and holes from the substrate, in a process assisted by the Coulomb field attraction from the previously stored carriers. Note that during the second carrier injection, a sizeable percentage of the trapped charge tunnels back to the substrate, and therefore, the achievement of an efficient emission depends on both the retention time of the first carrier injected and the tunnelling time of the incoming second carrier. Due to the lower barrier for electrons, the migration of the stored charge is more marked when holes are injected into electron-charged Si-nc, which results in a weaker emission compared to the case of electron injection into hole-charged nanocrystals[36]. This behaviour is clearly seen in figure $15(\mathrm{a})$, where the EL intensity of the peaks differs in approximately one order of magnitude. The hole-triggered peak is slightly faster in duration, even though the difference with the peak associated with the electron injection into hole-charged Si-nc does not appear to be significant. In any case, both transients are faster than those observed under optical excitation. The emission onset is particularly fast compared to PL rise times and gradually increases with the Si excess (from $\sim 0.5 \mu \mathrm{s}$ to $\sim 1 \mu \mathrm{s}$ ). Figure 15(b) depicts the emission decays as a function of the Si excess. These transients are characterized by stretched exponentials with typical time constants from $\sim 5 \mu$ s to $\sim 30 \mu$ s, with $\beta \sim 0.5$. The plot shows a progressive increase of the de-excitation time that peaks at a Si concentration of $13.7 \%$ (PD4). Beyond this point the EL decay becomes faster probably due to the activation of non-radiative Auger processes. The absorption-cross section associated to these processes can be now calculated by applying the following equation:

$$
\frac{1}{\tau_{\text {rise }}^{E L}}=\frac{1}{\tau_{E L}}+\sigma_{a b s}^{E L} \cdot \Phi_{J}
$$

that is analogous to the equation obtained for photo-excitation [equation 7]. The average current flux is represented by $\Phi_{J}$ and is estimated from the $\mathrm{I}-\mathrm{V}$ characteristics. The application of this equation leads to absorption-cross sections of $\sim 10^{-11} \mathrm{~cm}^{-2}$, approximately three orders of magnitude larger than values usually reported under DC excitation[31]. However, some authors have pointed out[32, 33] that the typically reported values of $\left(\sim 10^{-14} \mathrm{~cm}^{-2}\right)$ must be interpreted as a lower bound since leakage currents not contributing to the emission are being taken into account unavoidably in the calculations. Therefore, our results should be considered as the result of a strong reduction of the leakage current (thanks to the AC polarization) that leads to a better profit of the injected charge. Furthermore, in reference [32], Carreras et al. estimated an absorption-cross section of about $10^{-12} \mathrm{~cm}^{-2}$ that is close to our results. Figures 15(b) and 15(c) compare the PL emission of sample P2 with the EL spectrum of device PD3 (both structures have similar Si contents). The spectral range clearly links the EL emission to $\mathrm{Si}-\mathrm{nc} / \mathrm{SiO}_{2}$ systems, even though the peak position is slightly blue-shifted and the full width at half maximum (FWHM) is narrower as compared 
to PL measurements. The difference is attributed to the modulation introduced by the polycrystalline $\mathrm{Si}$ electrode, which transmittance in the visible range is strongly distorted by interferences phenomena [see figure 15(b)]. In order to check this point, the EL spectrum is compared with the one obtained by combining the PL emission and the electrode transmittance. The good match obtained between both spectra observed in figure 15(c) underlines the important role played by the top contact on the emission properties. The spectral measurements in the NIR range reveal EL emission from the substrate at $\sim 1.1 \mathrm{eV}$, in agreement with the results obtained under optical pumping. The voltage at the emission onset and the resistivity of the active layer are represented in figure 16(a). Note how the increase of the layer conductivity facilitates the carrier injection and, consequently, the achievement of detectable emission at lower voltages.
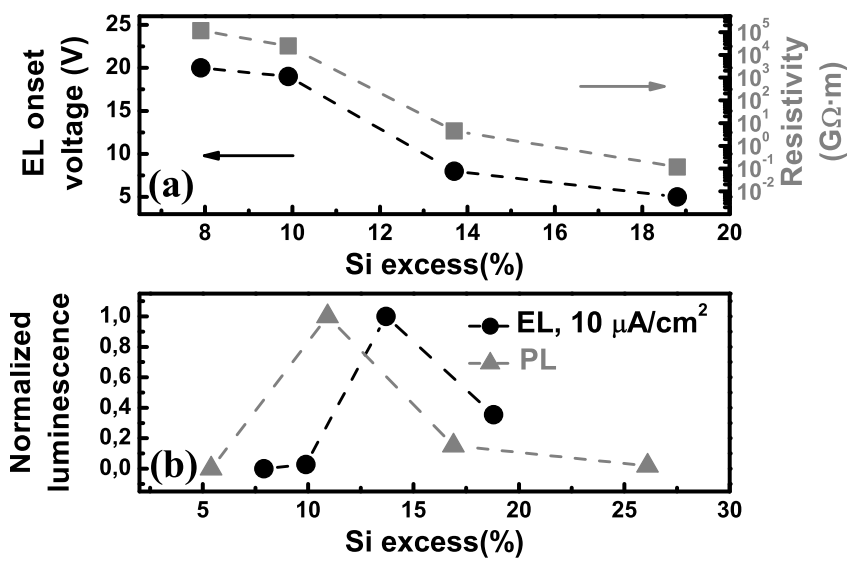

Figure 16. (a) Variation of the onset voltage and the resistivity of the active layers with the Si excess. Note that the resistivity is represented in log scale. (b) Evolution of the PL and EL intensities with the Si content.

The device breakdown also depends on the Si excess, as it is related with the current level through the device. The samples with large Si contents, PD5 and PD6, breakdown at about $14 \mathrm{~V}$ and $22 \mathrm{~V}$ respectively, whereas the samples with low Si concentrations do not present an observable breakdown, even for voltages higher than $30 \mathrm{~V}$. Figure 16(b) shows the EL and PL intensities as a function of the Si content. The EL corresponds to a fixed current density of $\sim 10 \mu \mathrm{A} / \mathrm{cm}^{2}$. In accordance with the quantum confinement theory (QCT), similar behaviours are observed, even though the EL intensity peaks at higher Si contents than in the case of the emission under optical pumping, $\sim 13.7 \%$ versus $\sim 11.2 \%$. This difference is due to the fact that under electrical pumping, an optimum emission requires an additional amount of $\mathrm{Si}$ in order to improve carrier injection.

\subsection{Ion implantation}

The characteristics of the different devices fabricated by ion implantation are presented in table 4. The Si contents were estimated from the XPS profiles of the test layers, 


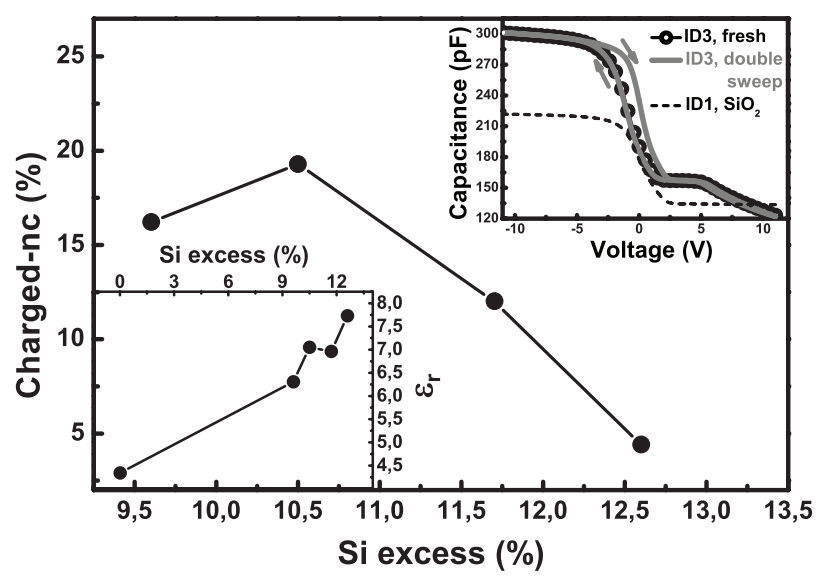

Figure 17. Ratio of charged-nc as a function of the Si execss. The upper inset represents an example of $\mathrm{C}-\mathrm{V}$ curves obtained from sample ID3. The black solid arrows indicate the sweep direction. In the lower inset, the values of $\epsilon_{r}$ are represented for the different devices (i.e, as a function of the Si excess).

taking into account the nominal implantation doses of tables 2 and 4 . The table also shows the thickness of the different active layers measured by ellipsometry that reveal a slight increase of thickness with the Si excess. C-V characteristics were measured in order to obtain the dielectric permittivity of the $\mathrm{SRO}, \epsilon_{r}$, [from $C_{\max }$ and equation 9] and the density of trapped charge, $N_{t}$. The upper inset of figure 17 shows a typical C-V curve of implanted devices. In general, the capacitance hysteresis is characterized by a sizeable trapping of electrons that is related to an easier carrier injection (if compared with PECVD), as it will be demonstrated below. The lower inset of the same figure shows the values of $\epsilon_{r}$ as a function of the $\mathrm{Si}$ excess. There is a good correlation between $\epsilon_{r}$ and the increase of the Si content. Note that the values of $\epsilon_{r}$, in general, are slightly higher than those of PECVD structures, probably due to an incomplete removal of the Si3N4 buffer used during the implantation process and/or to the fact that PECVD layers exhibit a higher porosity. The density of trapped charge is calculated from the capacitance hysteresis cycle with a similar procedure as in the case of PECVD samples. The calculations reveal a maximum value of $N_{t}$ in structures with low $\mathrm{Si}$ concentrations. In those structures (ID2 and ID3), typical densities of $\sim 1.0-1.5 \times 10^{12}$ $\mathrm{cm}^{-2}$ are observed that represent percentages of charged nanocrystals of about $16-19 \%$. As the Si concentration increases, the trapped charge progressively decreases (see figure 17), reaching a minimum value of $\sim 4 \times 10^{11} \mathrm{~cm}^{-2}$ in sample ID6, corresponding to an occupation percentage of $\sim 4.4 \%$. This is due to the increase of the conductivity in the SRO that favours the migration of charge. On the other hand, it is worth noting that, in implanted samples, the continuous distribution of Si-nc facilitates the carrier injection that now is achieved at relatively low voltages $(\sim 3-4 \mathrm{~V})$. Figure 18 shows the current-voltage curve of sample ID6. Implanted structures, in general, exhibit higher current levels than PECVD devices (for comparison see figure 14). Such behaviour is attributed to their continuous and uniform in-depth Si-nc distributions. The I-V curves 


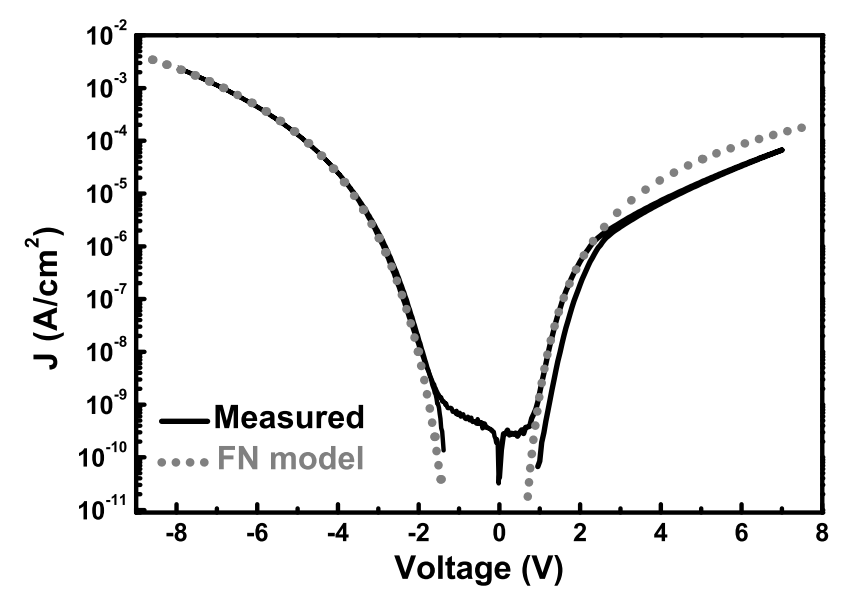

Figure 18. Current-voltage characteristics of sample ID6 is represented together with a FN fit. Note the fast increase of the current density with the applied voltage and the good agreement of the FN fits.

are well fitted by Fowler-Nordheim expressions which onsets are placed in the range of $\sim 2-3 \mathrm{~V}$, remarkably lower than those observed in deposited structures. As will be shown later, also in implanted devices the FN onset is closely related to the observation of a measurable EL emission. The effective energy barriers extracted from the fits are very low (about $0.2 \mathrm{eV}$ ) compared to those calculated for deposited structures, which is attributed to the continuous distribution of Si-nc through the SRO. EL emission analysis is represented in figure 19: figure 19(a) shows the time-resolved EL of structure ID4 under a 9-V square wave voltage and figure 19(b) depicts the EL spectrum of each device. Time-resolved measurements reveal two different contributions to the electroluminescence. The first corresponds to field-effect luminescence generated by the sequential injection of electrons and holes. The second contribution is a sizeable DC contribution observed during the negative half-cycle that, for a driving frequency of $\sim 1$ $\mathrm{kHz}$, is approximately one order of magnitude higher than the field-effect luminescence. This component, not observed in deposited devices, is linked to the continuous Si-nc distribution and the consequent high current levels observed in figure 18. The inset of figure 19(b) compares the average current through the SRO layer with the intensity of the DC emission, both magnitudes given as a function of the driving voltage. The good accordance between both magnitudes suggests that impact ionization is the mechanism responsible of the DC emission. While it is true that bipolar injection from opposite electrodes could be also possible, it is less probable due to the thickness of the layers. It should be noted that the idea of simultaneous carrier injection from substrate and gate electrodes could perfectly explain the DC component observed, as it is only present during the negative half-cycle of the gate bias.

The transients of the DC contribution are approximately one order of magnitude longer than those of the field effect emission. Onset and decay times slightly increase with the Si content reaching values of $0.5 \mathrm{~ms}$ and $0.7 \mathrm{~ms}(\beta \sim 0.5)$ in sample ID6, 

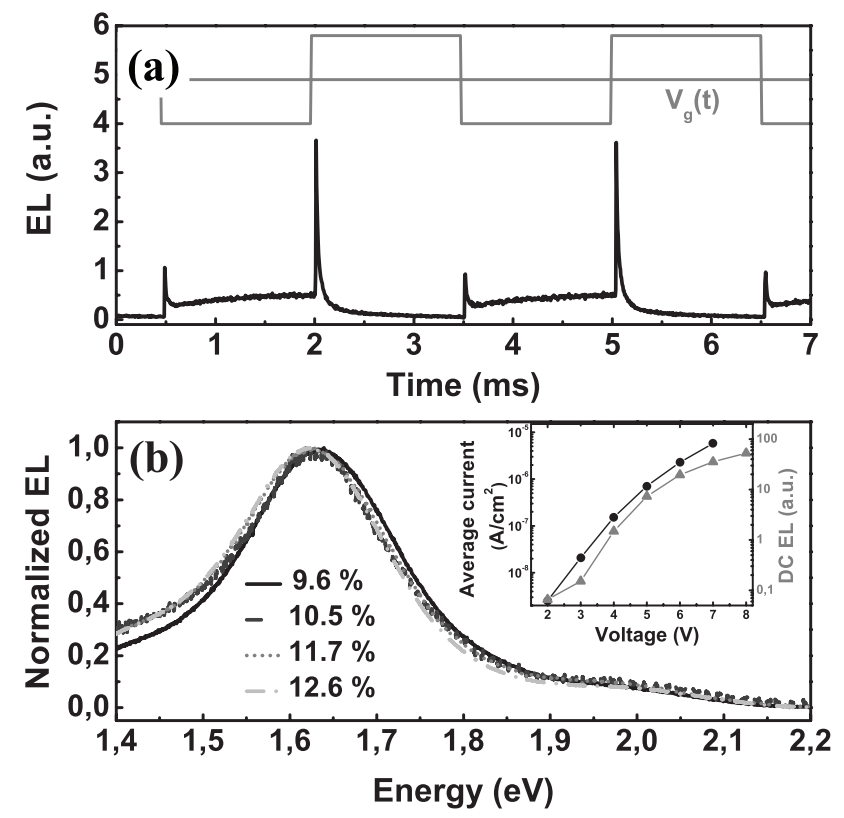

Figure 19. (a) Time-resolved EL of sample ID4 under $9 \mathrm{~V}$ square waves. (b) EL spectra of the different implanted structures. In the inset, the average current and the DC EL emission of sample ID6 are represented as functions of the gate voltage.

respectively. The calculation of the $\mathrm{DC}$ onset requires the suppression of the $\mathrm{AC}$ transient that can be made by merely exciting with negative pulses, as shown in reference[12]. The DC decay was extracted by modelling the de-excitation at the rise edge of the gate bias with two exponentials: the first one corresponding to the fast $\mathrm{AC}$ transient and the second one, associated with the continuous emission. From equation 12 it is possible to calculate the absorption cross section of the DC mechanism. The value of was found around $3 \times 10^{-14} \mathrm{~cm}-2$, in agreement with values usually reported from Si-nc based devices under DC polarization[31].

With respect to the field-effect transients, it is clearly seen in figure 19(a) that the electron-triggered emission is larger in intensity than that observed when holes are injected into electron charged Si-nc, even though the difference between these two peaks is not as significant as in deposited structures (due to some extent by the higher conductivity of the layer). The transient times of both peaks are very similar and slightly increase with the Si concentration. The onsets are typically placed in the range of $\sim 5-8 \mu$ s whereas the de-excitation approximately is in the range of $\sim 20-30 \mu$ s. This emission decay is extracted from a stretched exponential fit with typical $\beta$ of about 0.5 . By combining these results with equation 12, typical absorption cross sections of about $2 \times 10^{-12} \mathrm{~cm}^{2}$ are obtained, approximately two orders of magnitude higher than that for the DC emission. This difference suggests that the field-effect mechanism takes best profit of the injected charge as compared to the impact ionization mechanism. Note that in PECVD samples the value of ??? is one order of magnitude higher, which is attributed to their relatively low operation currents. 


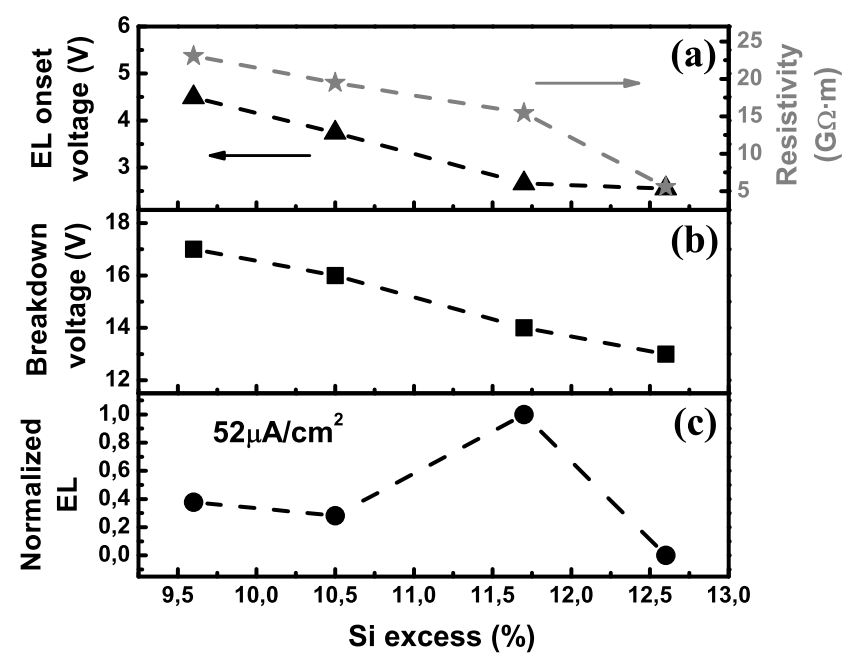

Figure 20. (a) Variation of the onset voltage and the resistivity of implanted structures. (b) Breakdown voltage of the same set of samples also as a function of the Si content. (c) Dependence of the EL intensity from these devices on the added Si.

Figure 19(b) shows the EL spectra of the different implanted devices under 9$\mathrm{V}$ square wave voltages. In contrast with photoluminescence, the electroluminescence is independent of the $\mathrm{Si}$ content. Nevertheless, this apparent effect is essentially due to the modulation induced by the interference phenomena inside the poly-Si layer, as discussed above. No emission has been observed in the NIR range from bulk-Si bandto-band transitions, in agreement with PL results. Figure 20(a) compares the EL onset voltage and layer resistivity as a function of the Si-excess. As can be seen, the addition of Si reduces the resistivity of the SRO layer favouring the carrier injection and shifting the onset voltage to lower values. Nevertheless, this leads to higher current flows that unavoidably lead to lower breakdown voltages, as shown in figure 20(b). The integrated EL was studied in the different structures for a given current flow of $\sim 52 \mu \mathrm{A} / \mathrm{cm}^{2}$. The dependence of the emission on the $\mathrm{Si}$ excess is very similar to the results presented in figure 16(b), and is in good agreement with the QCT. The maximum emission is observed in device ID5 (11.7\% of Si excess).

\section{LEDs performances}

In order to study the suitability of PECVD and implanted devices as candidates for a $\mathrm{Si}$ nanocrystal-based LED implementation, we compare in this section the best operation performances of these structures. Figure 21(a) shows the response of the field-effect luminescence of samples ID5 and PD3 (with similar Si contents) to different driving frequencies. First, the curves show that as the integration time is held constant, enhancement of the EL signal is initially observed due to the increasing number of integrated cycles[36]. The EL intensity peaks at a frequency that is determined by both the tunneling dynamics and recombination times. As shown in the plot, the 

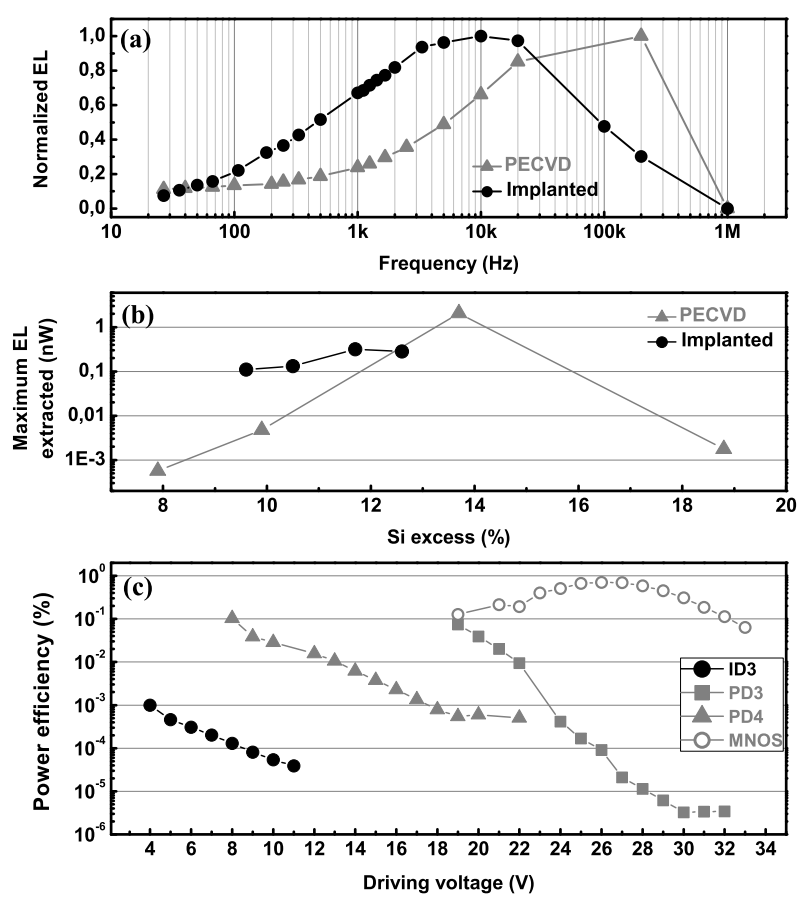

Figure 21. (a) EL response of samples ID5 and PD3 against the driving frequency. (b) Maximum output power extracted from the different samples. (c) Variation of the emission power efficiency with the driving voltage.

shorter transient times observed in PECVD structures allow a faster modulation of the emission compared with implanted samples. Figure 21(b) compares the maximum EL intensity achievable for PECVD and implanted samples. To obtain this value, the devices were biased at its optimum frequency [see figure 21(a)] with amplitudes close to the breakdown voltage (if any). Implanted devices exhibit typical emission powers of $\sim 0.1-0.3 \mathrm{nW}$. The slight increase observed with the $\mathrm{Si}$ content is related to the higher conductivity of the layers and consequently, mostly due to larger contributions of the DC mechanism. Note that the quantum confinement modulation of the emission seen in figure 20(c) is not observed, which is due to the fact that EL intensities were calculated at different pump fluxes (the maximum before breakdown). With respect to PECVD structures, the most intense emission is observed in sample PD4 with a maximum output power of $\sim 2 \mathrm{nW}$ (higher than those in implanted samples) that contrasts with the low values of the rest of the deposited structures that exhibit maximum output powers lower than $0.01 \mathrm{nW}$. By comparing these results with the ratio of charged nanocrystals represented in figure 13(c), it becomes clear that for low $\mathrm{Si}$ contents (samples PD2 and PD3), the poor emission obtained is due to a deficient carrier injection into the Si-nc, as only a small part of the Si-nc distribution can be excited ( $\sim 0.4 \%$ and $\sim 8.9 \%$, respectively). In contrast, for samples PD4 and PD5 the ratio of excitable Si-nc is remarkably higher $(\sim 90 \%)$, but only in the first case intense emission is observed. The weak emission of sample PD5 is, in contrast to samples PD2 and PD3, 
associated to the loss of quantum confinement and to the emergence of carrier migration phenomena. Figure 21(c) compares the power efficiency of different deposited and implanted samples to the values obtained from a reference device, with a MNOS (metal nitride oxide semiconductor) configuration that has been recently reported as a highefficiency structure[34]. A thin buffer of pure $\mathrm{Si}_{3} \mathrm{~N}_{4}(\sim 15 \mathrm{~nm})$ is deposited onto the SRO in order to limit the device leakage current. More details about this kind of structures can be found in reference [34]. The values depicted in figure 21(c) are calculated by adjusting the gate voltage to its optimum frequency and under the assumption that the emission pattern of these devices is approximately Lambertian. The implanted samples present maximum values of efficiency of $\sim 10^{-3 \%}$ that rapidly decrease with the applied voltage as the increasing power consumption is not compensated by the gain in the output power. Thanks to the low current levels observed in PECVD samples, their values of power efficiency are typically two orders of magnitude higher than that observed in implanted structures, up to $\sim 0.1 \%$. Also in this case, a fast reduction of the efficiency with the applied voltage is observed, mostly due to the strong increase of the FN current at higher fields. In the case of the MNOS structure, the nitride buffer reduces the device leakage current leading to a better profit of the injected charge that translates into power efficiencies of about $1 \%$ and output emission powers of $\sim 10 \mathrm{nW}$. It is worth noting the slow variation of the power efficiency with the gate bias in MNOS structures that is attributed to the increase of the FN onset[34].

Based on these results, it is clear that, in order to achieve efficient devices, the fabrication of discontinuous Si-nc distributions appears to be beneficial, as it reduces the device power consumption. On the other hand, if we take into account the ratios of charged nanocrystals represented in figure 13(c) and figure 17, it is also clear that, apart from the reduction of the power consumption, the power efficiency can be improved by increasing the number of excited Si-nc. This should be done by accurately controlling the amount of defects and the size and position of the Si-nc distribution. In the light of our results, the combination of these two factors could enhance the power efficiency of Si-nc based MOS devices up to $\sim 1-10 \%$ with typical powers in the range of $\sim 10-20$ $n W$.

Implanted and PECVD structures were submitted to accelerated aging for more than $24 \mathrm{~h}$ of continuous operation under application of $13 \mathrm{~V}$ and $30 \mathrm{~V}$ square waves, respectively, at a frequency of $1 \mathrm{kHz}$. Figure 22 shows the degradation of the emission for samples PD4 and ID6. The plot shows that sample ID6 exhibits an emission degradation rate of about $45 \%$ that contrasts with the lower value observed in sample PD4, about 20\%. This difference correlates with the respective current levels observed in I-V characteristics. Both curves are well fitted by a logarithmic function within the experimental timescale[35], resulting in estimated lifetimes of several months. 


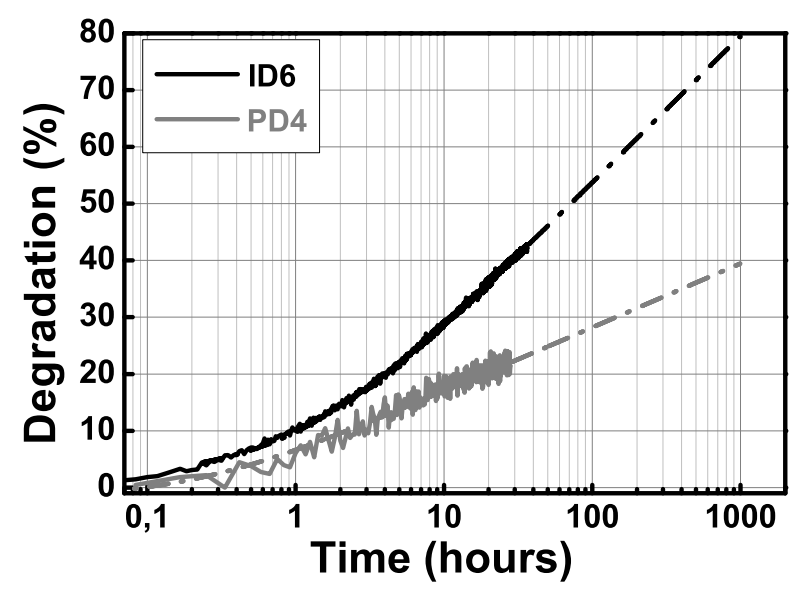

Figure 22. Degradation of the emission in structures ID6 and PD4 as a function of the operation time.

\section{Conclusions}

The luminescence of $\mathrm{Si}-\mathrm{nc} / \mathrm{SiO}_{2}$ layers fabricated by PECVD and ion implantation has been thoroughly studied under optical and electrical excitation. The results have been correlated with an exhaustive characterization of the structural and electrical properties. First, XPS and EFTEM measurements revealed that, in spite of the almost flat profile of Si excess, PECVD layers exhibited a $\sim 5-\mathrm{nm}$ Si-nc free region, attributed either to the diffusion of Si to the substrate or to the instabilities at the first stages of the deposition process. In implanted layers, the profile of added Si is also relatively flat (XPS) and translates into a uniform and continuous Si-nc distribution (EFTEM), which clearly underlines the suitability of the implantation process. We have also shown that for similar Si contents, the average size of the Si-nc obtained by ion implantation are smaller than those observed in deposited layers, which leads to a higher Si-nc density and shorter interdot (interaction range) distances. The PL emission showed a good agreement between PECVD and implanted samples with maximum intensities of emission for layers with $\sim 11 \%$ of Si excess. In both cases, the absorption cross sections are of the order of $10^{-16} \mathrm{~cm}^{2}$.

EL measurements have been performed by polarizing with bipolar and monopolar square wave voltages. The EL spectra of implanted and PECVD structures reveal typical emission from $\mathrm{Si}-\mathrm{nc} / \mathrm{SiO}_{2}$ systems that correlates with $\mathrm{PL}$ results. A strong modulation of the spectral shape and peak position is observed due to interferences phenomena in the poly-Si top contact. The time-resolved EL revealed both AC and DC emission from implanted samples, in contrast to the measurements carried out in PECVD devices, where only transient emission was observed. The absence emission under constant electrical excitation in the deposited layers is due to the discontinue Sinc distribution that hinders the DC flow through the structure. Moreover, this current reduction results in onset and breakdown voltages remarkably higher than in the case of 
implanted samples. The absorption cross sections of the implanted AC and DC emission are of the order of $\sim 10^{-12} \mathrm{~cm}^{2}$ and $\sim 10^{-14}$, several orders of magnitude higher than those values obtained by photoexcitation. In the case of PECVD structures the value of is approximately $\sim 10^{-11} \mathrm{~cm}^{2}$. The maximum of the EL intensity is observed in structures with Si contents between 11-13\%, apparently higher than that under optical excitation. We have also shown the limited frequency response of gate-biased Si-nc based MOS structures, attributed to the characteristic radiative lifetimes of the Si nanoparticles. The maximum EL intensities obtained are $\sim 0.3 \mathrm{nW}$ for implanted devices and $\sim 2$ $\mathrm{nW}$ for PECVD. The efficiency of implanted devices is of about $10^{-3} \%$, two orders of magnitude lower than the one obtained for PECVD structures, $0.1 \%$. This result tells that, in terms of efficiency, a continuous distribution of Si-nc appears to be detrimental.

In order to evaluate the room available to further enhance the emission capabilities in these LEDs, a rough estimation of the electrically active Si-nc (percentage of charged nanocrystals) has been performed. In all cases, most of the Si-ncs were not contributing to the emission, which power could be enhanced up to $\sim 20 \mathrm{nW}$, with typical power efficiencies in the range of $1-10 \%$.

Finally, accelerated aging studies reveal stronger degradation of the emission in implanted devices, which is attributed to their higher current levels in comparison to PECVD devices.

\section{Acknowledgments}

This work has been partially supported by the project MILES-SILUZ: TEC200613907/MIC, financed by the Spanish Ministry of Education and Science. Mr. A. Morales and Mr. J. Barreto acknowledge the grants received from CONACyT-FC and CSIC (I3P), respectively. Mr. M. Perlvarez acknowledges Dr. O. Jambois, R. RodrguezTrujillo, Y. Berencn, A. Santos, and Dr. J. Arbiol.

\section{References}

[1] Carreras J, Arbiol J, Garrido B, Bonafos C and Montserrat J 2008 Direct modulation of electroluminescence from silicon nanocrystals beyond radiative recombination rates Applied Physics Letters 92091103

[2] Jaiswal S L, Simpson J T, Withrow S P, White C W and Norris P M 2003 Design of a nanoscale silicon laser Applied Physics a-Materials Science and Processing 77 57-61

[3] Jalali B and Fathpour S 2006 Silicon photonics Journal of Lightwave Technology 24 4600-15

[4] Pavesi L, Dal Negro L, Mazzoleni C, Franzo G and Priolo F 2000 Optical gain in silicon nanocrystals Nature 408 440-4

[5] Prakash G V, Cazzanelli M, Gaburro Z, Pavesi L, Iacona F, Franzo G and Priolo F 2002 Nonlinear optical properties of silicon nanocrystals grown by plasma-enhanced chemical vapor deposition Journal of Applied Physics 91 4607-10

[6] Redding B, Creazzo T, Marchena E, Shouyuan S and Prather D W 2008 Design, fabrication, and characterization of a $1 D$ microcavity for enhanced luminescence from silicon nanocrystals 2008 5th IEEE International Conference on Group IV Photonics (GFP) 329-31 
[7] Rong H S, Jones R, Liu A S, Cohen O, Hak D, Fang A and Paniccia M 2005 A continuous-wave Raman silicon laser Nature 433 725-8

[8] De la Torre J, Souifi A, Poncet A, Busseret C, Lemiti M, Bremond G, Guillot G, Gonzalez O, Garrido B, Morante J R and Bonafos C 2003 Optical properties of silicon nanocrystal LEDs Physica E-Low-Dimensional Systems and Nanostructures 16 326-30

[9] DiMaria D J, Kirtley J R, Pakulis E J, Dong D W, Kuan T S, Pesavento F L, Theis T N, Cutro J A and Brorson S D 1984 Electroluminescence studies in silicon dioxide films containing tiny silicon islands Journal of Applied Physics 56 401-16

[10] Irrera A, Iacona F, Franzo G, Boninelli S, Pacifici D, Miritello M, Spinella C, Sanfilippo D, Di Stefano G, Fallica P G and Priolo F 2005 Correlation between electroluminescence and structural properties of Si nanoclusters Optical Materials 27 1031-40

[11] Luterova K, Pelant I, Valenta J, Rehspringer J L, Muller D, Grob J J, Dian J and Honerlage B 2000 Red electroluminescence in $\mathrm{Si}^{+}$-implanted sol-gel-derived $\mathrm{SiO}_{2}$ films Applied Physics Letters $\mathbf{7 7}$ $2952-4$

[12] Peralvarez M, Garcia C, Lopez M, Garrido B, Barreto J, Dominguez C and Rodriguez J A 2006 Field effect luminescence from Si nanocrystals obtained by plasma-enhanced chemical vapor deposition Applied Physics Letters 893

[13] Walters R J, Bourianoff G I and Atwater H A 2005 Field-effect electroluminescence in silicon nanocrystals Nature Materials 4 143-6

[14] Hernandez S, Pellegrino P, Martinez A, Lebour Y, Garrido B, Spano R, Cazzanelli M, Daldosso N, Pavesi L, Jordana E and Fedeli J M 2008 Linear and nonlinear optical properties of Si nanocrystals in $\mathrm{SiO}_{2}$ deposited by plasma-enhanced chemical-vapor deposition Journal of Applied Physics 103 064309-6

[15] Peralvarez M, Lopez M, Garrido B, Morante J R, Barreto J, Cadarso V J, Riera M, Zinoviev K and Dominguez C 2005 Precipitation of highly luminescent phases from PECVD Si suboxides Materials Research Society Symposium Proceedings 832 153-8

[16] Ziegler J F 2004 SRIM-2003 Nuclear Instruments and Methods in Physics Research Section BBeam Interactions with Materials and Atoms 219 1027-36

[17] Hernandez S, Martinez A, Pellegrino P, Lebour Y, Garrido B, Jordana E and Fedeli J M 2008 Silicon nanocluster crystallization in $\mathrm{SiO}_{x}$ films studied by Raman scattering Journal of Applied Physics 104

[18] Pai P G, Chao S S, Takagi Y and Lucovsky G 1986 Infrared spectroscopic study of SiO films produced by plasma enhanced chemical vapor-deposition Journal of Vacuum Science and Technology a-Vacuum Surfaces and Films 4 689-94

[19] Lucovsky G, Nemanich R J and Knights J C 1979 Structural interpretation of the vibrational spectra of -Si:H alloys Physical Review B 19 2064-73

[20] Garrido B, Lpez M, Garca C, Prez-Rodrguez A and Morante J R 2002 Influence of average size and interface passivation on the spectral emission of Si nanocrystals embedded in $\mathrm{SiO}_{2}$ Journal of Applied Physics 9110

[21] Belyakov V A, Burdov V A, Lockwood R and Meldrum A 2008 Silicon nanocrystals: fundamental theory and implications for stimulated emission Advances in Optical Technologies 279502 (32 pp.)

[22] Barreto J, Peralvarez M, Morales A, Garrido B, Montserrat J and Dominguez C 2008 Broad range adjustable emission of stacked SiNx/SiOy layers Journal of Materials Research 23 1513-6

[23] Delerue C, Allan G and Lannoo M 1993 Theoretical aspects of the luminescence of porous silicon Physical Review B 48 11024-36

[24] Matsumoto T, Suzuki J, Ohnuma M, Kanemitsu Y and Masumoto Y 2001 Evidence of quantum size effect in nanocrystalline silicon by optical absorption Physical Review B 63195322

[25] Takagahara T and Takeda K 1992 Theory of the quantum confinement effect on excitons in quantum dots of indirect-gap materials Physical Review B 46 15578-81

[26] Dufour C, Chausserie S and Gourbilleau F 2009 Silicon-rich/silica multilayers: A means to provide 
a link between the excitonic $1 D$ quantum confinement and the photoluminescence parameters $\mathrm{J}$. Lumines. 129 73-80

[27] Linnros J, Lalic N, Galeckas A and Grivickas V 1999 Analysis of the stretched exponential photoluminescence decay from nanometer-sized silicon crystals in $\mathrm{SiO}_{2}$ Journal of Applied Physics 86 6128-34

[28] Kovalev D, Heckler H, Polisski G and Koch F 1999 Optical properties of si nanocrystals Physica Status Solidi B-Basic Research 215 871-932

[29] Garcia C, Garrido B, Pellegrino P, Ferre R, Moreno J A, Morante J R, Pavesi L and Cazzanelli M 2003 Size dependence of lifetime and absorption cross section of Si nanocrystals embedded in $\mathrm{SiO}_{2}$ Applied Physics Letters 82 1595-7

[30] Garrido B, Cheylan S, Gonzalez-Varona O, Perez-Rodriguez A and Morante J R 2003 The effect of additional oxidation on the memory characteristics of metal-oxide-semiconductor capacitors with Si nanocrystals Applied Physics Letters 82 4818-20

[31] Irrera A, Pacifici D, Miritello M, Franzo G, Priolo F, Iacona F, Sanfilippo D, Di Stefano G and Fallica P G 2002 Excitation and de-excitation properties of silicon quantum dots under electrical pumping Applied Physics Letters 81 1866-8

[32] Carreras J and Garrido B 2008 Modeling direct modulation dynamics in silicon nanocrystal light emitting transistors. In: Group IV Photonics, 2008 5th IEEE International Conference on, pp $326-8$

[33] Valenta J, Lalic N and Linnros J 2004 Electroluminescence of single silicon nanocrystals Applied Physics Letters 84 1459-61

[34] Peralvarez M, Carreras J, Barreto J, Morales A, Dominguez C and Garrido B 2008 Efficiency and reliability enhancement of silicon nanocrystal field-effect luminescence from nitride-oxide gate stacks Applied Physics Letters 92241104

[35] Cui Z, Liou J J and Yue Y 2003 A new extrapolation method for long-term degradation prediction of deep-submicron MOSFETs IEEE Transactions on Electron Devices 50 1398-401

[36] Walters R J, Carreras J, Feng T, Bell L D and Atwater H A 2006 Silicon nanocrystal field-effect light-emitting devices IEEE J. Sel. Top. Quantum Electron. 12 1647-56 